\title{
mTOR controls neurotoxic lysosome exocytosis in inflammatory reactive astrocytes
}

Brendan Rooney $^{1}{ }^{*}$, Kun Leng ${ }^{1,2,3}$, Frank McCarthy ${ }^{4}$, Indigo V.L. Rose ${ }^{5}$, Kari A. Herrington ${ }^{6}$, Sophie Bax ${ }^{4}$, Marcus Y. Chin ${ }^{7,8}$, Saeed Fathi ${ }^{3}$, Manuel Leonetti ${ }^{4}$, Aimee W. Kao ${ }^{7}$, Joshua E. Elias $^{4}$, Martin Kampmann ${ }^{1,4,9,10} \mathbb{I}$

${ }^{1}$ Institute for Neurodegenerative Diseases, University of California, San Francisco, San Francisco, CA, USA

${ }^{2}$ Biomedical Sciences Graduate Program, University of California, San Francisco, San Francisco, CA, USA

${ }^{3}$ Medical Scientist Training Program, University of California, San Francisco, San Francisco, CA, USA

${ }^{4}$ Chan Zuckerberg Biohub, San Francisco, CA, USA

${ }^{5}$ Neuroscience Graduate Program, University of California, San Francisco, San Francisco, CA, USA

${ }^{6}$ Center for Advanced Microscopy, University of California, San Francisco, San Francisco, CA, USA

${ }^{7}$ Memory and Aging Center, Department of Neurology, University of California, San Francisco, San Francisco, CA, USA

${ }^{8}$ Small Molecule Discovery Center, Department of Pharmaceutical Chemistry, University of California, San Francisco, San Francisco, CA, USA

${ }^{9}$ Weill Institute for Neurosciences, University of California, San Francisco, San Francisco, CA, USA

${ }^{10}$ Department of Biochemistry and Biophysics, University of California, San Francisco, San Francisco, CA, USA

* These authors contributed equally

II e-mail: martin.kampmann@ucsf.edu

\section{ABSTRACT}

Inflammatory reactive astrocytes lose homeostatic functions and can be neurotoxic, potentially contributing to neurodegenerative diseases. However, the underlying cell biological mechanisms are not fully understood. Here, we demonstrate that lysosomes are remodeled and alkalinized in inflammatory reactive astrocytes, and that lysosome exocytosis drives astrocyte-mediated neurotoxicity. CRISPRi screens uncover mTOR as a regulator of neurotoxic lysosome exocytosis. These results pinpoint lysosome remodeling and exocytosis in inflammatory reactive astrocytes as a potential therapeutic target. 


\section{MAIN}

Reactive astrocytes are prevalent in many neurodegenerative diseases ${ }^{1}$. Mounting evidence suggests that inflammatory reactive astrocytes contribute to disease progression by losing their normal homeostatic functions and secreting factors that are neurotoxic under certain conditions ${ }^{1-}$ ${ }^{4}$. However, the mechanisms that drive these functional changes in inflammatory reactive astrocytes remain unknown.

To identify the cellular pathways responsible for the functional changes that accompany inflammatory astrocyte reactivity, we re-analyzed our previously collected RNA-sequencing dataset of hiPSC-derived astrocytes (iAstrocytes) $)^{5}$ treated with the microglia-derived cytokines IL1 $\alpha$, TNF, and C1q (hereafter referred to as ITC) which induce inflammatory reactivity in vitro $^{3}$ (Fig. 1a). As expected, many genes and pathways previously associated with inflammatory astrocyte reactivity ${ }^{2,6,7}$ were upregulated after ITC treatment, including SERPINA3 and C3, along with cellular pathways involved in NF- $\kappa \mathrm{B}$ signaling, antigen presentation, and cellular response to cytokines (Supplementary Table 1). Interestingly, many genes encoding lysosome-localized proteins were downregulated after ITC treatment (Fig. 1b-c). These included many subunits of the vacuolar ATPase (V-ATPase) complex and lysosomal degradative enzymes, which coordinate the proper acidification of lysosomes and hydrolysis of autophagic substrates (Fig. 1c, Supplementary Table 1). We validated that many of these genes were also downregulated at the protein level in ITC-treated iAstrocytes via proteomics (Extended Data Fig. 1a,b, Supplementary Table 2). Further analysis of published RNA-sequencing datasets of hiPSC-derived reactive astrocytes confirmed that this lysosomal signature was broadly conserved across models of inflammatory reactivity (Fig. 1c), including those that use different differentiation protocols (Barbar et al. ) $^{4}$ and cytokine treatments (Perriot et al. $)^{8}$. We also noted that a small subset of genes encoding lysosome-localized proteins involved in antigen presentation and inflammation (e.g. CTSS, HLA family) were strongly upregulated across models (Fig. 1c).

To determine if this lysosome signature is associated with changes in lysosome levels and function in inflammatory reactive astrocytes, we quantified levels of the lysosomal marker LAMP2 by immunoblotting, and found no significant difference after ITC treatment (Fig. 1d). Similarly, quantification of the lysosomal markers LAMP1 and LAMP2 by immunofluorescence did not show significant changes in response to ITC (Fig. 1e). However, there was a dramatic decrease in the fluorescent signal of LysoTracker Green, a cell-permeable, $\mathrm{pH}$-dependent dye that accumulates in lysosomes (Fig. 1e). We hypothesized that ITC-treated iAstrocytes were deficient in lysosomal acidification, thereby preventing LysoTracker from accumulating in lysosomes. Using the genetically encoded lysosome $\mathrm{pH}$ sensor FIRE-pHLy ${ }^{9}$, we found that the lysosomes of ITC-treated iAstrocytes were indeed less acidic than those of control iAstrocytes (Fig. 1f). Furthermore, ITC-treated iAstrocytes accumulated puncta of LC3B, an autophagosome marker degraded in the lysosome by acid-activated hydrolases (Fig. 1e). Using a GFP-LC3-RFP$\mathrm{LC} 3 \Delta \mathrm{G}$ reporter ${ }^{10}$, we verified that this accumulation in ITC-treated iAstrocytes reflected an impairment in degradative autophagic flux rather than an increase in the steady-state level of autophagic components (Fig. 1g). 
To gain a more detailed understanding of lysosome remodeling in inflammatory reactive astrocytes, we investigated the lysosomal proteome by immunoprecipitating a LAMP1-3xHA lysosome tag (lyso-IP) ${ }^{11}$ introduced into iAstrocytes via lentiviral transduction (Fig. 1h, Extended Data Fig. 1c,d). In agreement with our RNA-sequencing and whole-cell proteomics data, we found that many proteins required for lysosomal degradation, including V-ATPase subunits and lysosomal hydrolases, were less abundant in the lyso-IP fraction of ITC-treated iAstrocytes compared to vehicle-treated iAstrocytes (Fig. 1i, Supplementary Table 2). We also found that CST3, a potent inhibitor of lysosomal proteinases, was highly enriched in the lyso-IP fraction of ITC-treated iAstrocytes. In addition to the above signature of decreased degradative capacity, we found that several mediators of inflammatory signaling - CCL2, TNFAIP3, and IL32 - were more enriched in the lyso-IP fraction compared to whole-cell proteomics in ITCtreated vs. vehicle-treated iAstrocytes (Fig. 1j, Supplementary Table 2). Given that IL32 has previously been reported to undergo likely lysosome-mediated secretion ${ }^{12}$ and that many proteins traditionally involved in exocytosis (eg. RAB11A, RAB27A, RAB3A) were enriched in the lysoIP fraction upon ITC treatment (Fig. 1i,j, Supplementary Table 2), we hypothesized that inflammatory reactive astrocytes execute lysosome exocytosis, potentially as a mechanism to counteract the accumulation of autophagic substrates and/or as part of a non-canonical secretory pathway.

To test this hypothesis, we used TIRF microscopy to visualize lysosomes in iAstrocytes loaded with LysoTracker Green and expressing a lysosome membrane-targeted mCherry construct (Fig. 2a). Indeed, likely lysosome exocytosis events were observed as the trafficking of mCherry+/LysoTracker+ vesicles to the plasma membrane followed by an uncoupling of the mCherry and LysoTracker signals upon contact with the plasma membrane, after which the LysoTracker signal rapidly diffused, while the mCherry signal remained at the cell surface, suggesting the ejection of lysosomal contents (Fig. 2a-b, Supplemental Movies 1,2).

As an alternative and scalable approach to quantify lysosome exocytosis, we adopted a published flow cytometry-based protocol ${ }^{13,14}$ in which we stained non-permeabilized iAstrocytes with a fluorophore-conjugated antibody targeting a luminal epitope of LAMP1 that is exposed to the extracellular environment upon lysosome exocytosis (Extended Data Fig. 2a). With this approach, we found that ITC-treated iAstrocytes had almost twice the amount of cell-surface LAMP1 relative to vehicle-treated controls, despite equivalent levels of total LAMP1 protein between the two conditions (Fig. 2c,d). To test our hypothesis that the increased lysosome exocytosis in ITC-treated iAstrocytes was related to their deficit in lysosomal acidification, we treated iAstrocytes with bafilomycin A, a small molecule inhibitor of the V-ATPase complex. We observed a dose-dependent increase in lysosome exocytosis and concomitant decrease in lysosome acidification with increasing concentration of bafilomycin A (Fig. 2e). Together, these results suggest that lysosome remodeling involving decreased acidification and degradative capacity is associated with lysosome exocytosis in inflammatory reactive astrocytes.

Next, we turned our attention to understanding if lysosome exocytosis is involved in the known deleterious functions of inflammatory reactive astrocytes. Given that lysosome exocytosis contributes to the astrocyte secretome, we reasoned that lysosome exocytosis may play a role in the neurotoxicity of inflammatory reactive astrocytes. To test this hypothesis, we transferred astrocyte conditioned media (ACM) from vehicle-treated and ITC-treated iAstrocytes to hiPSC- 
derived neurons ${ }^{15}$ (iNeurons), and subsequently assayed the levels of both lysosome exocytosis (measured by cell-surface LAMP1 in the astrocytes) and neurotoxicity (measured using caspase 3/7 activity reporter dye in the ACM-receiving iNeurons) (Fig. 2f). Consistent with previous reports $^{3,4}$, the ITC-induced increase in cell-surface LAMP1 was mirrored by a strong increase in apoptosis in iNeurons receiving ACM derived from ITC-treated astrocytes (Fig. 2g,h). When we treated astrocytes with vacuolin-1, a small molecule inhibitor of lysosome exocytosis, there was a dose-dependent decrease in both lysosome exocytosis in astrocytes and apoptosis in iNeurons (Fig. 2g,). Importantly, unconditioned media supplemented with ITC or vacuolin-1 did not substantially affect apoptosis in iNeurons, ruling out the possibility that residual amounts of these compounds were causing the apoptosis effects in ACM experiments (Extended Data Fig. $2 b)$. In addition, vacuolin-1 did not induce excess cell death in iAstrocytes, ruling out the possibility that decreased astrocyte viability could explain the blunted neurotoxic activity (Extended Data Fig. 2c). Furthermore, to confirm that vacuolin-1 specifically inhibited lysosome exocytosis in astrocytes and did not perturb general vesicular trafficking to the plasma membrane, we co-stained astrocytes with an antibody against TFRC, a constitutively expressed plasma membrane receptor. We found no substantial effect of vacuolin-1 on cell-surface TFRC levels (Extended Data Fig. 2d). Lastly, we knocked down of genes reported to mediate lysosome exocytosis (SYT11 and VAMP7) ${ }^{16,17}$. While SYT11 and VAMP7 knockdown resulted in only a small decrease in cell-surface LAMP1, this was sufficient to reduce ITC-induced neurotoxicity by $\sim 50 \%$ relative to control iAstrocytes (Figure $2 \mathrm{i}, \mathrm{j}$ ).

To test if increasing lysosome exocytosis would also increase astrocyte-mediated neurotoxicity, we treated iAstrocytes receiving vehicle or ITC with a low dose of bafilomycin A (10 nM) to induce lysosome alkalinization and lysosome exocytosis, and transferred the conditioned media to iNeurons as before. Bafilomycin A treatment resulted in a robust increase in cell-surface LAMP1 in both astrocytes treated with ITC or vehicle (Fig. 2k). Astrocytes treated with ITC and bafilomycin A were approximately twice as neurotoxic compared to astrocytes treated with ITC and DMSO (Fig. 21). Interestingly, although bafilomycin A itself induced roughly the same level of lysosome exocytosis in vehicle-treated iAstrocytes as ITC-treated iAstrocytes, it did not cause a corresponding increase in neurotoxicity for iAstrocytes that were not treated with ITC, suggesting that ITC treatment alters the content of lysosomes in a way that increases neurotoxicity of lysosomal exocytosis. Neither ITC nor bafilomycin A (10 nM) induced apoptosis in iAstrocytes (Extended Data Fig. 2e) or iNeurons (Extended Data Fig. 2f). In summary, the above results suggest that lysosome exocytosis is a major mechanism underlying neurotoxicity of inflammatory reactive astrocytes.

To identify potential therapeutic targets to inhibit the lysosome alkalinization-exocytosisneurotoxicity axis in inflammatory reactive astrocytes, we performed targeted CRISPRi screens in iAstrocytes (Fig. 3a). Briefly, we transduced iAstrocytes expressing CRISPRi machinery ${ }^{5}$ with a lentiviral sgRNA library targeting the "druggable genome" $"$. After treating with ITC or vehicle control, we stained iAstrocytes with either LysoTracker or a fluorophore-conjugated LAMP1 antibody to monitor lysosomal alkalinization and exocytosis, respectively, and used fluorescence-activated cell sorting (FACS) to collect sgRNA-transduced astrocytes with low or high levels of LysoTracker or LAMP1. We then conducted next-generation sequencing to identify sgRNAs that were over- or under-represented within their respective cell population relative to non-targeting control (NTC) sgRNAs (see Methods). Based on this analysis, we 
identified candidate regulators (FDR $<0.05$, see Methods) of lysosomal $\mathrm{pH}$ (and also potentially mass; LysoTracker screen) or lysosome exocytosis (LAMP1 screen). Complete screen results are provided in Supplementary Table 3.

In agreement with our hypothesis that lysosomal alkalinization drives lysosome exocytosis, we found a strong inverse correlation between the LysoTracker and LAMP1 phenotypes for many hit genes (Fig. 3b, Extended Data Fig. 3). Across all screens, genes involved in mTOR signaling were highly enriched among hit genes, including MTOR itself as one of our strongest hits (Fig. $3 \mathrm{c}, \mathrm{d})$. To validate our screening results, we transduced iAstrocytes with an sgRNA targeting MTOR or a non-targeting sgRNA. As expected, MTOR knockdown dramatically increased LysoTracker staining (Fig. 3e). Importantly, MTOR knockdown specifically decreased lysosome exocytosis in ITC-treated but not vehicle-treated iAstrocytes (Fig. 3f), suggesting that mTOR activity may control lysosome exocytosis specifically in inflammatory reactive astrocytes. Consistent with our finding that suppressing lysosome exocytosis prevents inflammatory reactive astrocyte neurotoxicity, MTOR knockdown in iAstrocytes also rescued the neurotoxicity of ITCtreated iAstrocytes (Fig. 3g).

mTOR is a member of both mTORC1 and mTORC2 complexes, which regulate their downstream targets via phosphorylation ${ }^{19}$. Given that MTOR knockdown specifically blocked the ITC-induced increase of cell-surface LAMP1, we hypothesized that there would be increased mTOR activity in inflammatory reactive astrocytes. Immunoblots revealed that there were no significant changes in the levels of mTOR itself, but a clear trend towards increased phosphorylation of mTORC1 targets (p-S6 S235/236 and p-ULK1 S757) and to a lesser extent mTORC2 targets (p-Akt S473) in ITC-treated iAstrocytes (Fig. 3h-1).

To validate that mTOR activity is responsible for lysosomal changes in inflammatory reactive astrocytes using an orthogonal approach, we treated iAstrocytes with several mTOR inhibitors, including the pan-mTOR inhibitor PP242, in combination with vehicle and ITC. We found that PP242 suppressed mTOR activity (Fig. 3h), acidified lysosomes (Fig. 3m), decreased cellsurface LAMP1 (Fig. 3n), and blocked ITC-induced neurotoxicity (Fig. 3o) in a dose-dependent manner.

In summary, we uncovered lysosome remodeling - consisting of increased lysosome $\mathrm{pH}$, altered lysosomal content, and increased lysosome exocytosis - as a novel functional phenotype of inflammatory reactive astrocytes and a key pathological process mediating their neurotoxic activity. Furthermore, we used pooled CRISPRi screens to identify mTOR as a central upstream regulator of the lysosome alkalinization-exocytosis-neurotoxicity axis in inflammatory reactive astrocytes (Fig. 3p).

Our model is consistent with recent findings by other groups: First, in vivo reports that Lamp1 is the most strongly upregulated astrocyte cell-surface protein in a mouse model of multiple sclerosis (MS) ${ }^{20}$. Second, the fact that mTOR inhibition blunts disease progression in mouse models of amyotrophic lateral sclerosis ${ }^{21}$ and $\mathrm{MS}^{22}$ that are driven by inflammatory reactive astrocytes. Last, in vitro data suggesting a role for astrocyte lysosome exocytosis in HIV-Tatmediated neurotoxicity ${ }^{23}$. Mechanistically, we have not yet resolved if the relationship between lysosome exocytosis and astrocyte-mediated neurotoxicity stems from direct toxicity of astrocyte 
lysosome contents, or whether these contents contribute to autocrine-paracrine signaling in astrocytes to induce neurotoxicity through an indirect mechanism (Fig. 3p).

We hope that future work will bring clarity to how lysosome remodeling in astrocytes influences neurodegenerative diseases, particularly with respect to the disease-, region-, and contextspecific functions of reactive astrocytes in vivo. Given our in vitro results, we envision that these studies could inform novel therapeutic approaches.

\section{ACKNOWLEDGEMENTS}

We thank Greg Mohl, Avi Samelson, Olivia Teter, Biswa Ramani, Brandon Desousa, Emmy Li, Vukasin Jovanovic, Zuzana Krejciova, and Nawei Sun for contributions to preliminary studies and discussions. We thank members of the Kampmann lab for discussions and feedback on the manuscript. This research was supported by NIH grants F30 AG066418 to K.L., T32 NS115706 to I.V.L.R., R01 AG057342 and P30 AG062422 to A.W.K., and R01 AG062359 to M.K., a Chan Zuckerberg Initiative Award to A.W.K., and a Chan Zuckerberg Initiative Ben Barres Early Career Acceleration Award to M.K.

\section{AUTHOR CONTRIBUTIONS}

B.R., K.L., and M.K. conceptualized and led the overall project, and wrote the manuscript with input from all co-authors. B.R. performed the majority of experiments and data analysis. K.L developed the iAstrocyte platform, performed preliminary studies, and conducted the RNA-seq experiments. In consultation with J.E., F.M aided with sample preparation for whole-cell and lysosome-specific proteomics experiments, and performed mass spectrometry and preliminary computational analysis. I.V.L.R. performed sample prep for the cell-surface LAMP1 CRISPRi screens. K.A.H. guided TIRF experiments. M.L. and S.B aided with design and sample preparation for whole-cell and lysosome-specific proteomics experiments, respectively. M.Y.C. and A.W.K provided FIRE-pHLy reagents and guidance on lysosome $\mathrm{pH}$ experiments. S.F conducted LAMP2 immunofluorescence experiments and data analysis.

\section{COMPETING INTERESTS STATEMENT}

A.W.K is a member of the Scientific Advisory Board for Nine Square Therapeutics, Inc. J.E. is an advisor to Seer, Inc. M. K. has filed a patent application related to CRISPRi and CRISPRa screening (PCT/US15/40449) and serves on the Scientific Advisory Board of Engine Biosciences, Casma Therapeutics, and Cajal Neuroscience, and is an advisor to Modulo Bio and Recursion Therapeutics. None of the other authors declare competing interests.

\section{DATA AVAILABILITY STATEMENT}


Bulk RNA-seq data of hiPSC-derived astrocytes analyzed in this study were generated previously ${ }^{5}$ and are available on the Gene Expression Omnibus (GEO) under accession code GSE182307. Mass spectrometry results are in the process of submission to PRIDE.

\section{CODE AVAILABILITY STATEMENT}

The code used to analyze the data presented in this study will be shared upon request.

\section{METHODS}

\section{hiPSC culture}

Human iPSCs (male WTC11 background ${ }^{24}$ ) were cultured in Essential 8 (E8) Medium (ThermoFisher Scientific cat. no. A1517001) on BioLite Cell Culture Treated Dishes (ThermoFisher Scientific) coated with Growth Factor Reduced, Phenol Red-Free, LDEV-Free Matrigel Basement Membrane Matrix (Corning cat. no. 356231) diluted 1:100 in DMEM/F12 (ThermoFisher Scientific cat. no. 11330032). Essential 8 Medium was replaced daily. When hiPSC colonies demonstrated mature morphology, the hiPSCs were either clump passaged with EDTA for routine maintenance or dissociated to a near single-cell suspension with Accutase Cell Dissociation Reagent (ThermoFisher Scientific cat. no. A11105-01) for applications requiring cell counting. For clump passaging with EDTA, hiPSCs were washed with Dulbecco's phosphate buffered saline (DPBS; Milipore Sigma cat. no. D8537) and then incubated with Versene (ThermoFisher Scientific cat. no. 15040066) for 5-7 min at room temperature; the Versene solution was then aspirated and replaced with E8 + 10 nM Y-27632 dihydrochloride ROCK inhibitor (Tocris cat. no. 125410); hiPSC colonies were then gently detached mechanically using a cell scraper, resuspended gently, and passaged at 1:10-1:30 dilution in E8 + Y-27632, with Y27632 removed the next day. For near single-cell dissociation, hiPSCs were washed with DPBS, incubated with Accutase for $5-10 \mathrm{~min}$ at $37^{\circ} \mathrm{C}$, and then gently triturated with a $\mathrm{P} 1000$ pipette tip; the cell suspension was then diluted with PBS, collected into conical tubes, and spun down at $300 \mathrm{~g}$ for $3 \mathrm{~min}$; hiPSCs were then resuspended in E8 + Y-27632, counted, and plated onto Matrigel-coated plates at the desired density in E8 + Y-27632; Y-27632 would be maintained until the hiPSC colonies reached the appropriate size (> 40 cells). Studies with hiPSCs at UCSF were approved by the The Human Gamete, Embryo and Stem Cell Research (GESCR) Committee. Informed consent was obtained from the human subject when the WTC11 line was originally derived $^{24}$.

\section{Generation of iAstrocytes}

iAstrocytes were generated as detailed in Leng et al. ${ }^{5}$. Briefly, we generated hiPSCs (male WTC11 background) used for iAstrocyte differentiation by inserting a CRISPRi machinery cassette (pC13N-dCas9-BFP-KRAB, Addgene plasmid no. 127968) into the CLYBL safe-harbor locus and a doxycycline-inducible NFIA and SOX9 cDNA cassette into the AAVS1 safe-harbor 
locus using TALEN-based editing. After recovering a monoclonal CRISPRi-NFIA-SOX9 line, we differentiated hiPSCs to the neuroectoderm lineage via an embryoid body (EB)-based neural induction protocol. After dissociating plating EB-derived cells, we selected and expanded those forming neural rosettes. We used fluorescence activated cell sorting (FACS) to enrich for pure CD133+/CD271- neural progenitor cell (NPC) populations for expansion.

For iAstrocyte differentiation, these NPCs were re-plated on 1:200 Matrigel-coated dishes and cultured with ScienCell Astrocyte Media (ScienCell Research Laboratories cat. no. 1801) + 2 $\mu \mathrm{g} / \mathrm{mL}$ doxycycline (Millipore Sigma cat. no. D9891) to initiate iAstrocyte differentiation. A full media change was completed every other day (maintaining doxycycline at $2 \mu \mathrm{g} / \mathrm{mL}$ ) until NPCs reached confluency, at which time they were dissociated with Accutase and split 1:10 onto new Matrigel coated dishes (or cryopreserved) for continued differentiation and expansion. Expansion was continued until day 20 (d20) of differentiation, yielding iAstrocytes. See Leng et $a l . .^{5}$ for full details of iAstrocyte hiPSC generation and differentiation protocols.

\section{Induction of inflammatory reactivity in iAstrocytes}

For all iAstrocyte experiments in this study, d20 iAstrocytes were plated at 20,000 cells $/ \mathrm{cm}^{2}$ on 1:200 Matrigel-coated BioLite Cell Culture Treated Dishes (ThermoFisher Scientific) in ScienCell Astrocyte Media (without doxycycline) on d0. Full media changes were completed on $\mathrm{d} 1, \mathrm{~d} 3$, and d5. On d5, media was supplemented with IL-1 $\alpha$ (3 ng/mL; Peprotech cat. no. AF200-01A), TNF (30 ng/mL; Peprotech cat. no. AF-300-01A), and C1q (400 ng/mL; Complement Technology cat. no. A099) to induce inflammatory reactivity according to Liddelow et al. ${ }^{3}$. All experiments were conducted 24 hours after cytokine treatment (d6).

\section{CRISPRi-mediated gene knockdown using lentiviral transduction of individual sgRNAs}

To knock down genes with individual sgRNAs, we cloned CRISPRi sgRNAs into pMK1334 ${ }^{25}$ (Addgene cat. no. 127965), as previously described ${ }^{26}$. We generated lentivirus of the resulting constructs by co-transfecting them with $3^{\text {rd }}$ generation lentiviral packaging plasmids with TransIT-Lenti Transfection Reagent (Mirus cat. no. MIR6606) according to the manufacturer's protocol. The next day, lentivirus was precipitated using Lentivirus Precipitation Solution (ALSTEM cat. no. VC150) according to the manufacturer's protocol, resuspended in DPBS, and aliquoted and stored at $-80^{\circ} \mathrm{C}$. iAstrocytes were transduced with lentivirus at the time of plating. When necessary, the functional titer of the lentivirus was determined by measuring BFP+ cells 48 hours after transducing iAstrocytes with a serial dilution of lentivirus. The protospacer sequences of the sgRNAs used in this study were designed based on our next-generation CRISPRi algorithm ${ }^{18}$ and are listed below:

NTC - GAGACGAGGACATGTGTAGC

MTOR - GGGACAGCGGGGAAGGCGGG

SYT11 - GGAAGGGGGAAGGTATCCAG

VAMP7 - GAGGGACGCGGGTCAGTGCA 


\section{Generation and lentiviral transduction of reporter constructs}

To subclone the original FIRE-pHLy construct (pLBR10, Ubc:FIRE-pHLy) ${ }^{9}$ to make pLBR11 (GfaABC1D:FIRE-pHLy), we excised the FIRE-pHLy cassette from pLBR10 and linearized the backbone vector (pKL20; pHIV gfaABC1D:mRuby:WPRE) with BamH1-HF (New England BioLabs, cat. no. R3136) and ClaI (New England BioLabs cat. no. R0197). We then ran these reactions on a $1 \%$ agarose gel, gel extracted the relevant fragments, and ligated them together with T4 DNA Ligase (New England BioLabs cat. no. M0202) per manufacturer's protocol to generate pLBR11.

Both pLBR11 (all FIRE-pHLy experiments), and GFP-LC3-RFP-LC3 $\Delta \mathrm{G}$ (reporter cassette from Addgene plasmid \#84572) ${ }^{10}$ reporters were transduced into iAstrocytes with lentivirus at low multiplicity of infection (MOI) during plating to prevent high expression of exogenous lysosomal proteins.

\section{Immunofluorescence imaging}

For immunofluorescence imaging experiments, iAstrocytes plated in Greiner $\mu$ Clear 96-well plates (Greiner Bio-One cat. no. 655087) were fixed with 4\% paraformaldehyde (diluted from a $16 \%$ solution; Electron Microscopy Sciences cat. no. 15710) for $15 \mathrm{~min}$ at RT. After washing three times with DPBS, we blocked and permeabilized cells with DPBS (Milipore Sigma cat. no. D8537) + 3\% BSA (Milipore Sigma cat. no. A9647) + 0.1\% Triton X-100 (Millipore Sigma cat. no. X100) for 30 min at RT. Primary antibodies against LC3 (1:50; rabbit polyclonal; Cell Signaling Technology cat. no. 2775), LAMP1 (1:50; mouse monoclonal; Abcam cat. no. ab25630), or LAMP2 (1:500, Abcam cat. no. ab25631) were added to blocking buffer and incubated with iAstrocytes overnight at $4{ }^{\circ} \mathrm{C}$. Afterwards, the samples were washed with DPBS three times, incubated with pre-adsorbed secondary antibodies (1:500 goat anti-mouse IgG Alexa Fluor 488, 1:500 goat anti-rabbit IgG Alexa Fluor 555; Abcam cat. no. ab150119 and ab150086) for 1 hour at RT, washed three times with DPBS, incubated with $1 \mu \mathrm{g} / \mathrm{mL}$ Hoechst 33342 (ThermoFisher Scientific cat. no. H3570) and then washed two additional times before imaging on an IN Cell Analyzer 6000, using a 60X 0.7 NA objective, 2x2 binning, 100-400 ms exposure, an aperture width of $\sim 1$ Airy unit, and 9-16 fields per well.

\section{Lyso Tracker staining and imaging}

iAstrocytes were washed with DPBS and incubated with a $50 \mathrm{nM}$ solution of LysoTracker Green DND-26 (ThermoFisher Scientific cat. no. L7526) in DPBS for 5 minutes at $37^{\circ} \mathrm{C}$, washed two times with DPBS, and processed for downstream experiments. For imaging (Fig. 1e), iAstrocytes were imaged at $37^{\circ} \mathrm{C}$ and $5 \% \mathrm{CO}_{2}$ using an IN Cell Analyzer 6000 as above. For flow cytometry (CRISPRi screens), iAstrocytes were dissociated with Accutase for 10 mins at $37^{\circ} \mathrm{C}$ after LysoTracker staining, and resuspended in FACS buffer (DPBS + 1\% BSA + 2 mM EDTA (Milipore Sigma cat. no. 324506)).

\section{TIRF imaging}


iAstrocytes were plated at 20,000 cells $/ \mathrm{cm}^{2}$ in $35 \mathrm{~mm}$ imaging dishes (Ibidi, cat. no. 81156) and simultaneously transduced with pLBR11 (GfaABC1D:FIRE-pHLy). Prior to imaging, the conditioned media of iAstrocytes was collected. iAstrocytes were then stained with LysoTracker as detailed above and re-incubated with the collected conditioned media for the duration of imaging. TIRF was performed on a Nikon Ti-E inverted microscope equipped with a Nikon TIRF illuminator, Nikon motorized stage with piezo Z inset, Andor iXon Ultra DU897 camera, and run by NIS-Elements (v5.20.00 build 1423). iAstrocytes were imaged using an Apo TIRF 100x/1.49 oil (DIC N2/ 100X I) objective, 488nm and 561nm lasers (Agilent MLC400 monolithic laser combiner) and Sutter Lambda 10-B with ET525/50m and ET600/60m emission filters (Chroma), respectively, for 120 seconds with $100 \mathrm{~ms}$ exposure (per channel).

\section{Measurement of apoptosis in iNeurons treated with astrocyte-conditioned media}

hiPSC-derived neurons (iNeurons) were generated from WTC11 hiPSCs with a stably integrated dox-inducible NGN2 cassette (i3N iPSCs) as previously described ${ }^{15}$. Conditioned media was collected from iAstrocytes and spun down at $300 \mathrm{x}$ g for $5 \mathrm{~min}$ to remove dead cells, supplemented with CellEvent Caspase 3/7 Detection Reagent (ThermoFisher Scientific, cat. no. $\mathrm{C} 10423$ ) to a final concentration of $2 \mu \mathrm{M}$, and transferred to day 17 iNeurons after removing the original iNeuron media. As an unconditioned media control, we incubated ScienCell Astrocyte Media in identical conditions $\left(37^{\circ} \mathrm{C}, 5 \% \mathrm{CO}_{2}\right.$, cytokine and/or small-molecule compounds) as their respective experimental groups but without iAstrocytes. After a $\sim 24$ hour incubation period, iNeuron viability was assessed by imaging on an IN Cell Analyzer 6000, using a 20X 0.45 NA objective, $2 \times 2$ binning, environmental control set to $37{ }^{\circ} \mathrm{C}$ and $5 \% \mathrm{CO}_{2}$, an aperture width of $\sim 1$ Airy unit, $200 \mathrm{~ms}$ exposure, and 4-9 fields per well. The level of apoptosis in iNeurons was quantified relative to an unstained negative control with CellProfiler (see Data Analysis section).

\section{Antibody staining for flow cytometry}

To measure cell-surface protein levels (i.e. LAMP1, TFRC), iAstrocytes were dissociated with Accutase for 10 mins at $37{ }^{\circ} \mathrm{C}$, diluted with ice-cold DPBS $+1 \%$ BSA, and spun down at $300 \times \mathrm{g}$ for 5 minutes at $4{ }^{\circ} \mathrm{C}$. After aspirating the supernatant, all cells were incubated in 1:20 Fc block (BD Bioscience cat. no. 564220) diluted in DPBS + 3\% BSA for 10 minutes on ice, followed by the addition of 1:20 (1:40 final) primary antibodies against LAMP1 (AF488- or BV605conjugated; BioLegend cat. no. 328610 and 328634), and/or TFRC (FITC-conjugated, Biolegend, cat. no. 334104) for an additional 30 minute incubation on ice in the dark. iAstrocytes were then washed with ice-cold DPBS $+1 \%$ BSA, spun down at $300 \mathrm{x} \mathrm{g}$ for 5 mins at $4{ }^{\circ} \mathrm{C}$, and resuspended in DPBS $+1 \%$ BSA for flow cytometry.

\section{Pooled CRISPRi screening}

CRISPRi screens were conducted as described in Leng et al. ${ }^{5}$. Both LysoTracker and LAMP1 screens were conducted with the H1 (i.e. "druggable genome") sgRNA sub-library from our 
next-generation CRISPRi library ${ }^{18}$. We generated lentivirus for the pooled H1 sgRNA library as previously described $^{25}$. For each screen (i.e. LysoTracker or cell-surface LAMP1, vehicle or ITC), iAstrocytes were plated at 20,000 cells $/ \mathrm{cm}^{2}$ onto 4 Matrigel-coated $15-\mathrm{cm}$ dishes, transduced with the H1 lentiviral sgRNA library with $>70 \%$ transduction efficiency, treated with vehicle control or IL- $1 \alpha+\mathrm{TNF}+\mathrm{C} 1 \mathrm{q}$ for 24 hours, stained for LysoTracker or cell-surface LAMP1, and then sorted into LysoTracker high vs. low or cell-surface LAMP1 high vs. low (top and bottom $35 \%$ of cells on fluorescence histogram) populations. iAstrocytes were sorted with a BD FACSAria Fusion cell sorter at 5,000-10,000 events per second, and then pelleted via centrifugation at $300 \mathrm{x}$ g for 10 minutes for genomic DNA extraction. sgRNA abundances were then measured using next-generation sequencing as previously described ${ }^{25}$. LysoTracker screens were performed with two experimental replicates per condition, while LAMP1 screens were done with one replicate. Results from all CRISPRi screens in this study can be interactively explored at www.crisprbrain.org.

\section{Western blots}

iAstrocytes were dissociated with Accutase for 10 mins at $37^{\circ} \mathrm{C}$, spun down at $300 \mathrm{x} \mathrm{g}$ for 5 mins, washed with ice-cold DPBS, lysed in RIPA buffer (Thermo Fisher Scientific, cat. no. 89900) with protease (Roche, cat. no. 04693159001) and phosphatase inhibitors (Sigma-Aldrich, cat. no. PHOSS-RO), and incubated on ice for 45 minutes with brief vortexing every 15 minutes. Samples were centrifuged at $18,000 \mathrm{x}$ g for $20 \mathrm{mins}$ at $4^{\circ} \mathrm{C}$ to pellet insoluble components of lysate. After the total protein was quantified using the Pierce BCA Protein Assay Kit (ThermoFisher Scientific, cat. no. 23225) in the supernatant, each sample was diluted to the same concentration, and denatured with NuPAGE LDS Sample Buffer (4X) (ThermoFisher Scientific, cat. no. NP0007) and reduced with DTT.

Equivalent protein amounts (20-40 ug) were run on 10\% NuPAGE Bis-Tris gels (ThermoFisher Scientific, cat. no. NP0301) and transferred to nitrocellulose membranes (Bio-Rad, 1704271). We performed ponceau staining (Milipore Sigma, cat. no. P7170) to validate equivalent loading between lanes. Membranes were blocked with TBST (TBS + 0.1\% Tween-20) $+5 \%$ BSA for 1 hour at RT on a shaker, after which they were incubated with primary antibodies against phospho-S6 (1:500; polyclonal rabbit; Cell Signaling Technology cat. no. 2211), S6 (1:1000; monoclonal rabbit; Cell Signaling Technology cat. no. 2217), phospho-ULK1 (1:500; polyclonal rabbit; Cell Signaling Technology cat. no. 14202), phospho-Akt (1:500; monoclonal rabbit; Cell Signaling Technology cat. no. 4060), pan-Akt (1:1000; monoclonal mouse; Cell Signaling Technology cat. 2920), LAMP1 (1:1000; mouse monoclonal; Abcam cat. no. ab25630), LAMP2 (1:1000; mouse monoclonal; Abcam cat. no. ab25631), CTSD (1 ug/mL, R\&D Systems cat. no. AF1014), $\beta$-actin (1:1000, rabbit monoclonal; Cell Signaling Technology cat. no. 4970), or EGFP (1:200; mouse monoclonal; Santa Cruz Biotechnology cat. no. sc-9996) at $4^{\circ} \mathrm{C}$ overnight on a shaker. Blots were washed with TBS and incubated with secondary antibodies goat antimouse IRDye 800CW (1:10,000; LI-COR cat. no. 926-32210) and goat anti-rabbit IRDye 680RD (1:10,000; LI-COR cat. no. 926-68071) in blocking buffer (see above) for 1 hour at RT on a shaker. Afterwards, blots were washed with TBS, and imaged using an Odyssey Fc Imaging system (LI-COR, cat. no. 2800). To re-probe blots, we incubated membranes with $1 \mathrm{X}$ NewBlot Nitro Stripping Buffer (LI-COR, cat. no. 928-40030) for 5 minutes at RT on a shaker before re- 
blocking and probing as above. Immunoblot bands were quantified with LI-COR ImageStudio software.

Source data for immunoblots is provided in Supplementary Figures 2-5.

\section{Whole-cell proteomics sample preparation}

iAstrocytes were washed and collected (detached gently using a cell scraper) in ice-cold DPBS, spun down at $300 \mathrm{x}$ g for 5 mins at $4{ }^{\circ} \mathrm{C}$, and stored at $-80{ }^{\circ} \mathrm{C}$. Cell pellets were lysed in $2.5 \%$ sodium dodecyl sulfate (SDS) $50 \mathrm{mM}$ Tris $\mathrm{pH} 8$ and heated to $95^{\circ} \mathrm{C}$ for $5 \mathrm{~min}$, then subjected to probe sonication. Lysates were centrifuged at $14000 \mathrm{~g}$ for $15 \mathrm{~min}$ before protein quantification using BCA assay (Pierce, Thermo Fisher Scientific) according to manufacturer's instructions. Protein lysates were acetone precipitated by adding 5 volumes of ice-cold acetone, vortexed, incubated at $-20{ }^{\circ} \mathrm{C}$ for $1 \mathrm{hr}$ and pelleted by centrifugation at $21000 \mathrm{~g}$ and $4{ }^{\circ} \mathrm{C}$ for $15 \mathrm{~min}$. Protein pellets were resuspended in $1 \%$ sodium deoxycholate (SDC) $50 \mathrm{mM}$ EPPS pH 8.5 and reduced by incubating at $37{ }^{\circ} \mathrm{C}$ for 20 min with rotation in the presence of $5 \mathrm{mM}$ DTT. Protein was then alkylated by incubating in the dark at $37^{\circ} \mathrm{C}$ for $20 \mathrm{~min}$ in the presence of $25 \mathrm{mM}$ iodoacetamide before digestion overnight with LysC at an enzyme-to-protein ratio of 1:50 followed by $3 \mathrm{hr}$ digestion with trypsin at an enzyme-to-protein ratio of 1:50. Digestion was stopped by acidification to $1 \%$ trifluoroacetic acid (TFA) and placed on ice for 10min to promote precipitation of SDC. The SDC was then pelleted and removed by centrifugation at $21000 \mathrm{~g}$ for $10 \mathrm{~min}$. The supernatant containing acidified peptides were then de-salted on in-house made Stagetips $^{27}$ packed with polystyrene divinyl benzene reverse phase sulphonate solid phase extraction material (CDS Empore, Fisher Scientific) as previously published ${ }^{27}$. Stagetips were prepared by activating with $100 \%$ methanol, conditioning with $80 \%$ acetonitrile containing $0.1 \%$ TFA, equilibrated with $0.2 \%$ TFA, followed by sample loading, washing twice with $99 \%$ isopropanol containing $0.1 \%$ TFA, washing twice with $0.2 \%$ TFA and once with $0.1 \%$ FA. Peptides were eluted with $60 \%$ acetonitrile (ACN) containing $0.5 \%$ ammonium hydroxide. The desalted peptides were then flash frozen and dried by centrifugal evaporation.

\section{Lysosome-specific proteomics sample preparation}

pLBR09 (gfaABC1D: LAMP1-XTEN80-mEGFP-3XHA) used for lysosome immunoprecipitation was generated by subcloning pLBR08 (EF1 $\alpha$ : LAMP1-XTEN80-mEGFP3XHA). Briefly, pLBR08 was generated via Gibson assembly of PCR-amplified LAMP1 cDNA (from mTagRFP-T-Lysosomes-20 acquired from Nikon Imaging Center at UCSF, Addgene plasmid \#58022) and PCR-amplified XTEN80-mEGFP-3xHA immunoprecipitation tag with a linearized backbone generated from ClaI and BspDI (New England BioLabs cat. no. R0557) digestion of pKL017 (pHIV EF1a:Clover:WPRE). pLBR09 was then generated by linearizing pKL020 (pHIV gfaABC1D:mRuby:WPRE) and excising the LAMP1-XTEN80-mEGFP-3XHA cassette of pLBR08 via restriction digest with XbaI (New England Biolabs cat. no. R0145) and ClaI. The resulting fragments were run on a $1 \%$ agarose gel and gel extracted (Zymoclean Gel DNA Recovery Kit, cat. no. D4007). These fragments were ligated together with T4 DNA Ligase per manufacturer protocol. iAstrocytes were plated at $20,000 \mathrm{cells} / \mathrm{cm}^{2}$ per experimental 
replicate and transduced with pLBR09 lentivirus during plating. We used three replicates each for vehicle and ITC conditions.

Lysosome immunoprecipitation was performed as previously described ${ }^{11}$. Briefly, iAstrocytes were washed and collected (detached gently using a cell scraper) in ice-cold DPBS, spun down at $300 \mathrm{x}$ g for 5 mins at $4{ }^{\circ} \mathrm{C}$. We then resuspended the cell pellet in homogenization buffer ( 25 $\mathrm{mM}$ Tris $\mathrm{HCl} \mathrm{pH} 7.5,50 \mathrm{mM}$ sucrose, $0.5 \mathrm{mM} \mathrm{MgCl}_{2}, 0.2 \mathrm{mM}$ EGTA with protease inhibitors). The iAstrocyte cell suspension was mechanically lysed with a $23 \mathrm{G}$ syringe, immediately transferred to isotonic buffer (2.5M surcrose, $0.2 \mathrm{mM}$ EGTA, $0.5 \mathrm{mM} \mathrm{MgCl}$ ) and spun at 3000 $\mathrm{x} g$ for $10 \mathrm{mins}$ at $4{ }^{\circ} \mathrm{C}$. The supernatant was transferred to anti-HA magnetic beads (Thermo Scientific cat. no. PI88836) for the pulldown of intact lysosomes, with the final resuspension in $1 \mathrm{X}$ urea buffer (2M Urea, $1 \mathrm{mM}$ DTT, $12.5 \mathrm{mM}$ Tris). On-bead digest was performed by sequentially incubating the resulting protein samples with $5 \mathrm{mM}$ (final concentration) IAA at $22^{\circ} \mathrm{C}$ and $225 \mathrm{rpm}$ for $30 \mathrm{mins}, 0.5 \mathrm{ug}$ LysC at $22^{\circ} \mathrm{C}$ and $225 \mathrm{rpm}$ overnight, $1 \mathrm{ug}$ of trypsin at $22^{\circ} \mathrm{C}$ and $225 \mathrm{rpm}$ for 4 hours, and $1 \%$ (final concentration) TFA. Acidified peptides were then de-salted according to the same protocol as whole-cell proteome samples. For western blotting (Extended Data Fig. 1c), NuPAGE LDS Sample Buffer (4X) was added to samples collected prior to the on-bead digestion. See Western blots section for further details.

\section{LC-MS Analysis}

Peptides were resuspended in $2 \%$ ACN with $0.1 \%$ TFA before loading onto a $25 \mathrm{~cm}$ x $75 \mu \mathrm{m}$ ID, $1.6 \mu \mathrm{m} \mathrm{C} 18$ column (IonOpticks) maintained at $40^{\circ} \mathrm{C}$. Peptides were separated with an EASYnLC 1200 system (Thermo Fisher Scientific, San Jose, CA) at a flow rate of $300 \mathrm{nl} \mathrm{min}^{-1}$ using a binary buffer system of $0.1 \%$ FA (buffer A) and $80 \%$ acetonitrile with $0.1 \%$ FA (buffer B) in a two-step gradient. The following was performed for whole cell proteome samples, $3 \%$ to $27 \% \mathrm{~B}$ in $105 \mathrm{~min}$ and from $27 \%$ to $40 \% \mathrm{~B}$ in $15 \mathrm{~min}$. For lysosome-specific samples, $3 \%$ to $27 \% \mathrm{~B}$ in $52.5 \mathrm{~min}$ and from $27 \%$ to $40 \% \mathrm{~B}$ in $14.5 \mathrm{~min}$. All samples were analyzed on a Fusion Lumos mass spectrometer (Thermo Fisher Scientific, San Jose, CA) equipped with a nanoFlex ESI source operated at 1550 volts, RF lens set to $30 \%$, operated in data dependent acquisition mode with a duty cycle time of $1 \mathrm{sec}$. Full MS scans were acquired with a m/z scan range of 375-1500 $\mathrm{m} / \mathrm{z}$ in the Orbitrap mass analyzer (FTMS) with a resolution of 240k for whole cell proteome and $120 \mathrm{k}$ for lysosome-specific samples. Selected precursor ions were subjected to fragmentation using higher-energy collisional dissociation (HCD) with a quadrupole isolation window of 0.7 $\mathrm{m} / \mathrm{z}$, and normalized collision energy of $31 \%$. HCD fragments were analyzed in the Ion Trap mass analyzer (ITMS) set to Turbo scan rate. Fragmented ions were dynamically excluded from further selection for a period of $45 \mathrm{sec}$ for whole cell proteome and $60 \mathrm{sec}$ for lysosome-specific samples. The AGC target was set to 1,000,000 and 10,000 for full FTMS and ITMS scans, respectively. The maximum injection time was set to Auto for both full FTMS and ITMS scans.

\section{Drug treatments}

Vacuolin-1 (Milipore Sigma, cat. no. 673000), bafilomycin A1 (Milipore Sigma, cat. no. B1793), rapamycin (Milipore Sigma, cat. no. 553210), and PP242 (Milipore Sigma, cat. no. 475988) were resuspended per manufacturer's instructions, aliquoted, and stored at $-80^{\circ} \mathrm{C}$. All compounds 
were added to iAstrocytes alongside vehicle or ITC treatments (i.e. 24 hour treatments) with the exception of bafilomycin, which was added to cultures for the 2-4 hours preceding experiments for purposes of completely alkalinizing lysosomes.

\section{Data analysis}

\section{Analysis of published RNA-seq datasets}

We used BioJupies ${ }^{28}$ to reanalyze bulk RNA-seq data and obtain differentially expressed genes from Perriot et al. ${ }^{8}$ (GSE120411; hiPSC-derived astrocytes treated with IL1 $\beta$ and TNF) and Barbar et al. ${ }^{4}$ (syn21861229; CD49f+ astrocytes sorted from cerebral organoids treated with vehicle control or IL-1 $\alpha+\mathrm{TNF}+\mathrm{C} 1 \mathrm{q})$,

\section{Proteomics}

Raw files were searched against the human reference proteome (Swiss-Prot, downloaded on $20^{\text {th }}$ of April 2020, refer to PRIDE upload for full sequence) along with the default common contaminants list with enzyme specificity set to trypsin within MaxQuant version 2.0.1.0 using standard settings with match between runs and the MaxLFQ algorithm activated. Whole cell proteome (raw data file names begin with 'Proteome') and lysosome-specific (raw data file names begin with 'LAMP1') raw files were set as separate parameter groups to ensure that the MaxLFQ algorithm was performed only within each sample set. The resulting proteinGroups text file was then uploaded to Perseus version 1.6.7.0 where all reverse sequences, potential contaminants and proteins only identified by site were filtered out of the dataset. All protein abundance values were normalized by Log 2 transformation. The lysosome-specific raw data files were evaluated separate from whole cell proteomic files. Search results from lysosomal raw data files were filtered to include proteins that were quantified in at least two out of three biological replicates within at least the ITC (raw data file names include ITC) or vehicle (raw data file names include WT) triplicate set. Missing values were then imputed within each replicate by replacing from the normal distribution using default settings. A two sample students t-test was performed between the ITC treated and vehicle triplicates, with permutation-based FDR using default settings. The resulting T-test difference and significance were used to generate the lysosome-specific volcano plot.

\section{Pathway enrichment analysis}

We used Enrichr ${ }^{29-31}$ to perform enrichment analysis of gene lists.

\section{Flow cytometry}


Data from flow cytometry experiments were analyzed using FlowJo (version 10.7.1). Live cells were gated by plotting SSC-A vs. FSC-A and then single cells were gated by plotting FSC-H vs. FSC-A. For experiments involving CRISPRi knockdown, analysis was restricted to sgRNAtransduced cells (gating on the histogram of BFP fluorescence values). For antibody staining experiments where median fluorescence intensity (MFI) values were reported, the average MFI of unstained control samples were subtracted from the MFI of stained samples. An example gating strategy from the resulting flow cytometry data is show in Supplemental Figure 1.

\section{Fluorescence imaging analysis}

We used CellProfiler (v3.15) (2) $^{32}$ to quantify iAstrocyte immunostaining and neuron apoptosis (as measured by cleaved caspase 3/7 fluorescent dye) imaging data. For neuron viability, monochrome images of caspase $3 / 7$ were thresholded ("Threshold" module, "Manual" setting) with respect to unstained (no caspase 3/7 dye) controls. The caspase 3/7 signal was then quantified as the total intensity for each image, using the thresholded image as a mask. Similarly, for iAstrocyte fluorescence microscopy images, the total image intensity of LAMP1, LAMP2, LC3, and LysoTracker signals were quantified after first thresholding images relative to either a no-primary antibody control (immunofluorescence-LAMP1, LAMP2, LC3) or no-dye control (LysoTracker). The total image intensity of LAMP1, LAMP2, and LC3 was then divided by the total intensity of Hoechst to correct for variation in cell number. All frames collected were averaged to return a single quantification per well, which we considered as a single data point for statistical tests.

\section{Statistics and reproducibility}

Sample sizes were determined by referencing existing studies in the field. Major findings were validated using independent samples and orthogonal approaches. Numbers of replicates are listed in each figure. Randomization was not relevant to our study because no animals or human subjects were involved. Statistics were computed in RStudio (version 4.0.5). Correction for multiple testing was performed where appropriate.

\section{References}

1. Phatnani, H. \& Maniatis, T. Astrocytes in neurodegenerative disease. Cold Spring Harb Perspect Biol 7 (2015).

2. Escartin, C., et al. Reactive astrocyte nomenclature, definitions, and future directions. Nature neuroscience 24, 312-325 (2021).

3. Liddelow, S.A., et al. Neurotoxic reactive astrocytes are induced by activated microglia. Nature 541, 481-487 (2017). 
4. Barbar, L., et al. CD49f Is a Novel Marker of Functional and Reactive Human iPSCDerived Astrocytes. Neuron 107, 436-453 e412 (2020).

5. Leng, K., et al. CRISPRi screens in human astrocytes elucidate regulators of distinct inflammatory reactive states. bioRxiv 2021.08.23.457400 (2021).

6. Zamanian, J.L., et al. Genomic analysis of reactive astrogliosis. J Neurosci 32, 63916410 (2012).

7. Brambilla, R., et al. Inhibition of astroglial nuclear factor kappaB reduces inflammation and improves functional recovery after spinal cord injury. J Exp Med 202, 145-156 (2005).

8. Perriot, S., et al. Human Induced Pluripotent Stem Cell-Derived Astrocytes Are Differentially Activated by Multiple Sclerosis-Associated Cytokines. Stem cell reports 11, 11991210 (2018).

9. Chin, M.Y., et al. Genetically Encoded, pH-Sensitive mTFP1 Biosensor for Probing Lysosomal pH. ACS Sens 6, 2168-2180 (2021).

10. Kaizuka, T., et al. An Autophagic Flux Probe that Releases an Internal Control. Mol Cell 64, 835-849 (2016).

11. Abu-Remaileh, M., et al. Lysosomal metabolomics reveals V-ATPase- and mTORdependent regulation of amino acid efflux from lysosomes. Science 358, 807-813 (2017).

12. Hasegawa, H., Thomas, H.J., Schooley, K. \& Born, T.L. Native IL-32 is released from intestinal epithelial cells via a non-classical secretory pathway as a membrane-associated protein. Cytokine 53, 74-83 (2011).

13. Andrews, N.W. Detection of Lysosomal Exocytosis by Surface Exposure of Lamp1 Luminal Epitopes. Methods Mol Biol 1594, 205-211 (2017).

14. Rodriguez, A., Webster, P., Ortego, J. \& Andrews, N.W. Lysosomes behave as Ca2+regulated exocytic vesicles in fibroblasts and epithelial cells. J Cell Biol 137, 93-104 (1997).

15. Wang, C., et al. Scalable Production of iPSC-Derived Human Neurons to Identify TauLowering Compounds by High-Content Screening. Stem cell reports 9, 1221-1233 (2017).

16. Sreetama, S.C., Takano, T., Nedergaard, M., Simon, S.M. \& Jaiswal, J.K. Injured astrocytes are repaired by Synaptotagmin XI-regulated lysosome exocytosis. Cell Death Differ 23, 596-607 (2016).

17. Verderio, C., et al. TI-VAMP/VAMP7 is the SNARE of secretory lysosomes contributing to ATP secretion from astrocytes. Biol Cell 104, 213-228 (2012).

18. Horlbeck, M.A., et al. Compact and highly active next-generation libraries for CRISPRmediated gene repression and activation. Elife 5 (2016). 
19. Saxton, R.A. \& Sabatini, D.M. mTOR Signaling in Growth, Metabolism, and Disease. Cell 169, 361-371 (2017).

20. Sanmarco, L.M., et al. Gut-licensed IFNgamma(+) NK cells drive LAMP1(+)TRAIL(+) anti-inflammatory astrocytes. Nature 590, 473-479 (2021).

21. Granatiero, V., et al. Modulation of the IGF1R-MTOR pathway attenuates motor neuron toxicity of human ALS SOD1(G93A) astrocytes. Autophagy, 1-14 (2021).

22. Clark, I.C., et al. Barcoded viral tracing of single-cell interactions in central nervous system inflammation. Science 372 (2021).

23. Fan, Y. \& He, J.J. HIV-1 Tat Promotes Lysosomal Exocytosis in Astrocytes and Contributes to Astrocyte-mediated Tat Neurotoxicity. J Biol Chem 291, 22830-22840 (2016).

24. Miyaoka, Y., et al. Isolation of single-base genome-edited human iPS cells without antibiotic selection. Nat Methods 11, 291-293 (2014).

25. Tian, R., et al. CRISPR Interference-Based Platform for Multimodal Genetic Screens in Human iPSC-Derived Neurons. Neuron 104, 239-255 e212 (2019).

26. Gilbert, L.A., et al. Genome-Scale CRISPR-Mediated Control of Gene Repression and Activation. Cell 159, 647-661 (2014).

27. Rappsilber, J., Mann, M. \& Ishihama, Y. Protocol for micro-purification, enrichment, pre-fractionation and storage of peptides for proteomics using StageTips. Nat Protoc 2, 18961906 (2007).

28. Torre, D., Lachmann, A. \& Ma'ayan, A. BioJupies: Automated Generation of Interactive Notebooks for RNA-Seq Data Analysis in the Cloud. Cell Syst 7, 556-561 e553 (2018).

29. Chen, E.Y., et al. Enrichr: interactive and collaborative HTML5 gene list enrichment analysis tool. BMC Bioinformatics 14, 128 (2013).

30. Kuleshov, M.V., et al. Enrichr: a comprehensive gene set enrichment analysis web server 2016 update. Nucleic Acids Res 44, W90-97 (2016).

31. Xie, Z., et al. Gene Set Knowledge Discovery with Enrichr. Curr Protoc 1, e90 (2021).

32. McQuin, C., et al. CellProfiler 3.0: Next-generation image processing for biology. PLoS Biol 16, e2005970 (2018).

33. Krencik, R. \& Zhang, S.C. Directed differentiation of functional astroglial subtypes from human pluripotent stem cells. Nat Protoc 6, 1710-1717 (2011).

34. Tyanova, S., Temu, T. \& Cox, J. The MaxQuant computational platform for mass spectrometry-based shotgun proteomics. Nat Protoc 11, 2301-2319 (2016). 
bioRxiv preprint doi: https://doi.org/10.1101/2021.09.11.459904: this version posted September 12. 2021. The copvriaht holder for this preprint (which was not certified by peer review) is the author/funder, who has granted bioRxiv a license to display the preprint in perpetuity. It is made available under aCC-BY 4.0 International license.

35. Tyanova, S. \& Cox, J. Perseus: A Bioinformatics Platform for Integrative Analysis of Proteomics Data in Cancer Research. Methods Mol Biol 1711, 133-148 (2018). 
a

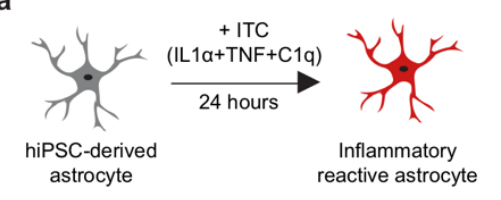

b

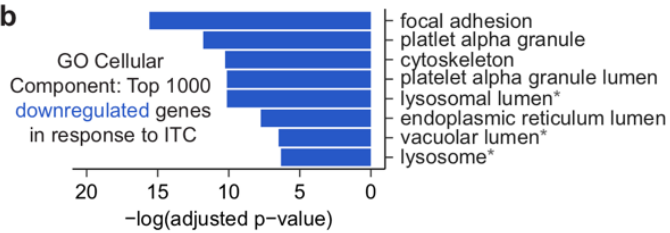

c

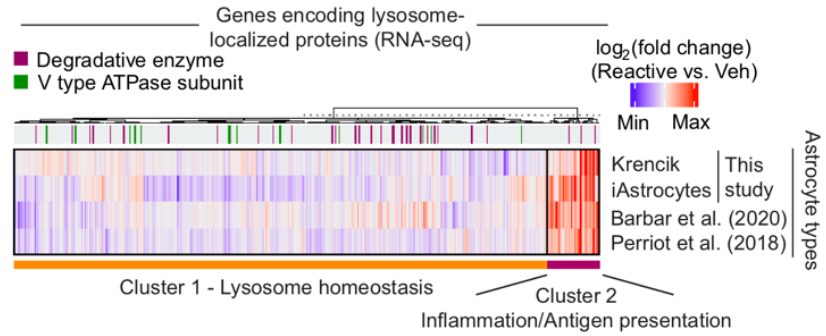

d
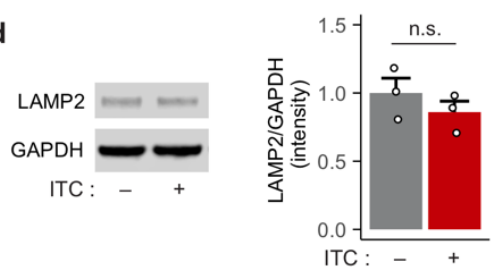

e

Inflammation/Antigen presentation
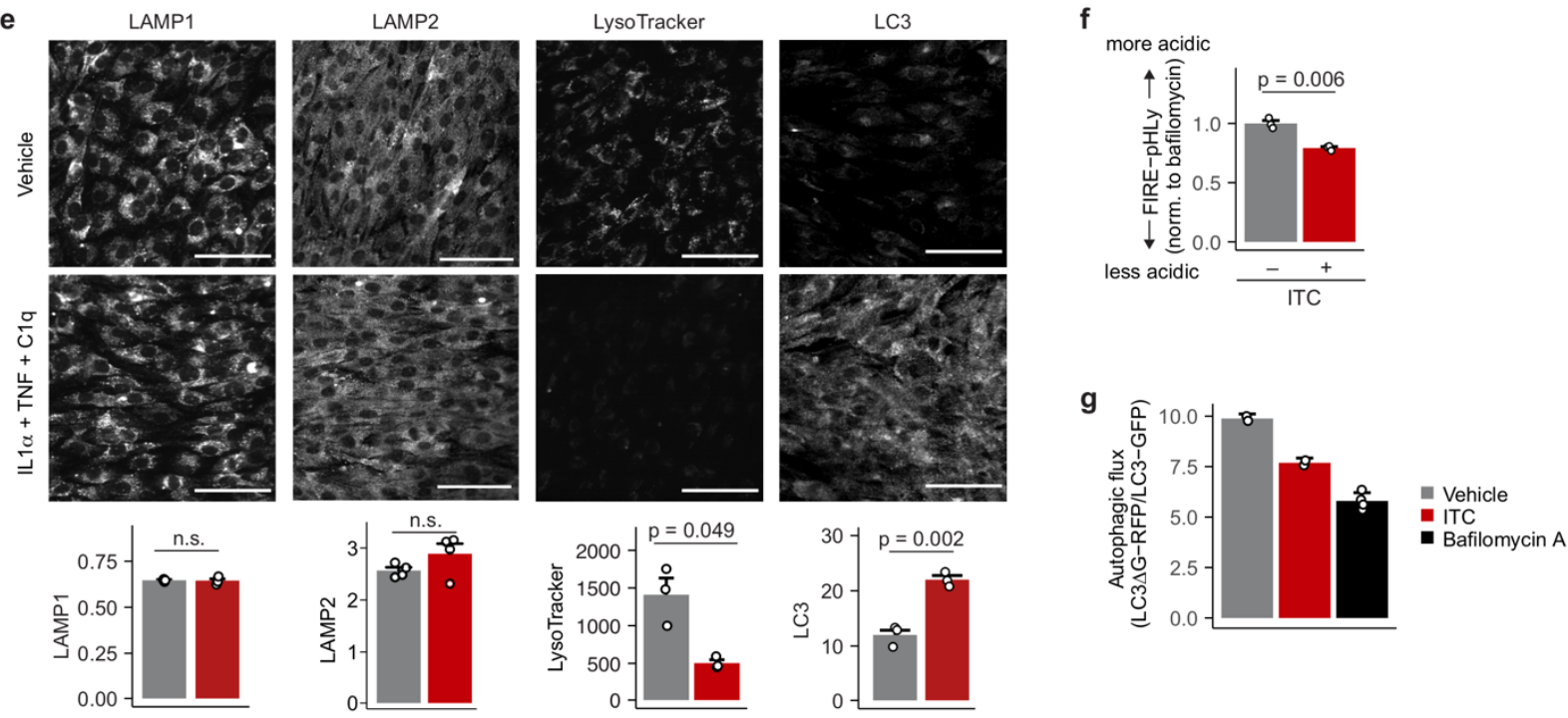

h

Lyso-IP strategy

Lysosome tagging via lentiviral transduction (pLBR09)
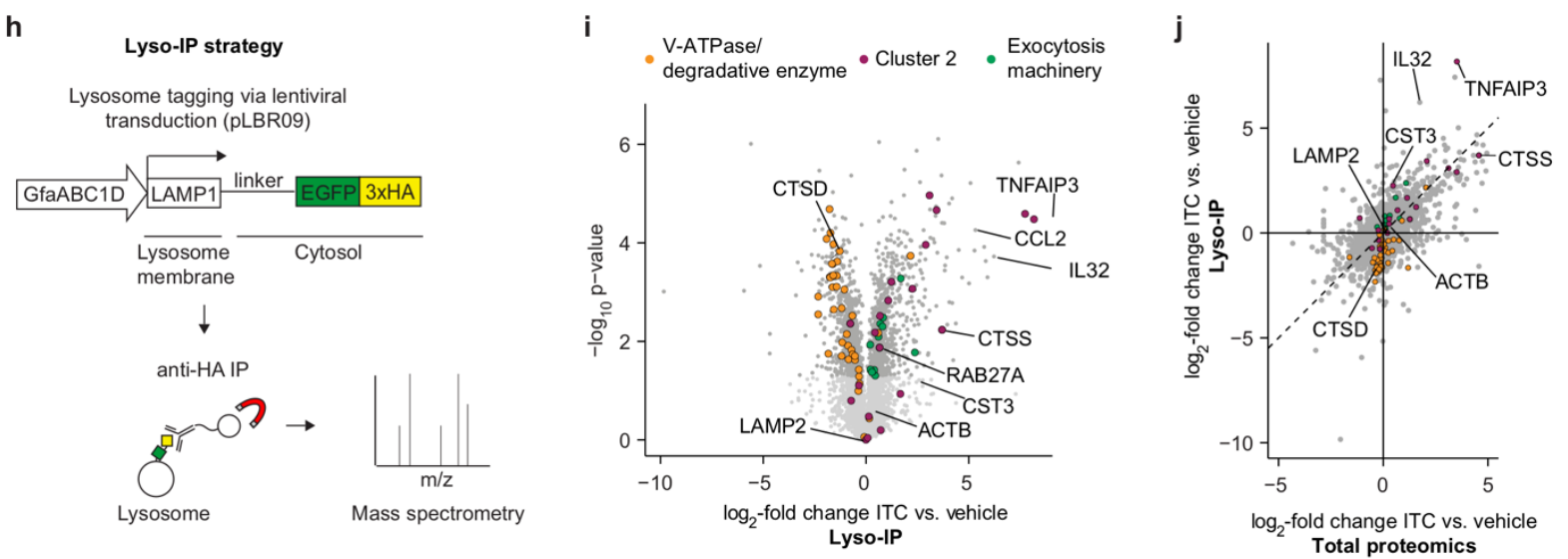

Fig. 1 | Lysosomes are remodelled in inflammatory reactive astrocytes. a, Schematic of the in vitro model of inflammatory astrocyte reactivity used in this study. b, Bar graph of Gene Ontology (GO) terms enriched among the top 1000 genes with decreased expression in iAstrocytes treated with IL1 $\alpha+\mathrm{TNF}+\mathrm{C} 1 \mathrm{q}$ (ITC) compared to vehicle control from RNA-Seq ( $\mathrm{n}=$ 
3 per condition). c, Heatmap of the mean $\log _{2}$ (fold change) of gene transcripts with GO annotations of lysosome, lysosomal membrane, or lysosomal lumen in response to inflammatory stimuli for astrocytes derived using protocols described in Leng et al. ${ }^{5}$ (iAstrocytes), Krencik et $a l .{ }^{33}$ and published datasets from Barbar et $a l .{ }^{4}$ and Perriot et al. ${ }^{8}$ (see Methods). d, left, representative immunoblot of total LAMP2 protein in iAstrocytes treated with ITC or a vehicle control; right, quantification of blots from $\mathrm{n}=3$ independent wells. e, Representative images of vehicle- (top) and ITC-treated (middle) iAstrocytes stained for the indicated lysosome/autophagosome markers, with corresponding quantification (bottom, see Methods; $\mathrm{n}=$ 3 independent wells per condition; scale bar $=75 \mu \mathrm{m}$ ). f, Lysosome acidity of FIRE-pHLyexpressing iAstrocytes (measured by flow cytometry) treated with ITC relative to vehicle-treated controls ( $n=3$ (for LAMP2, $n=4$ ) independent wells for each condition). g, Autophagic flux (measured with GFP-LC3-RFP-LC3 $\Delta$ G reporter-expressing iAstrocytes) treated with vehicle, ITC, or $100 \mathrm{nM}$ bafilomycin A ( $n=3$ independent wells for each condition). $\mathbf{h}$, Workflow of lysosome proteomics (lyso-IP, see Methods). i, Volcano plot of the $\log _{2}$ (fold change) and associated log-scaled $\mathrm{p}$-values of protein abundances detected by lyso-IP from astrocytes treated with ITC relative to vehicle-treated controls (see Methods; $n=3$ per condition). $\mathbf{j}$, Scatter plot of the $\log _{2}$ (fold change) of proteins detected by lyso-IP versus whole-cell proteomics from astrocytes treated with ITC relative to vehicle-treated controls (see Methods; $n=3$ per condition). For bar graphs, individual data points represent individual wells, while error bars represent the standard error of the mean (s.e.m.). The two-sided Student's t-test was used in panels d-f. 


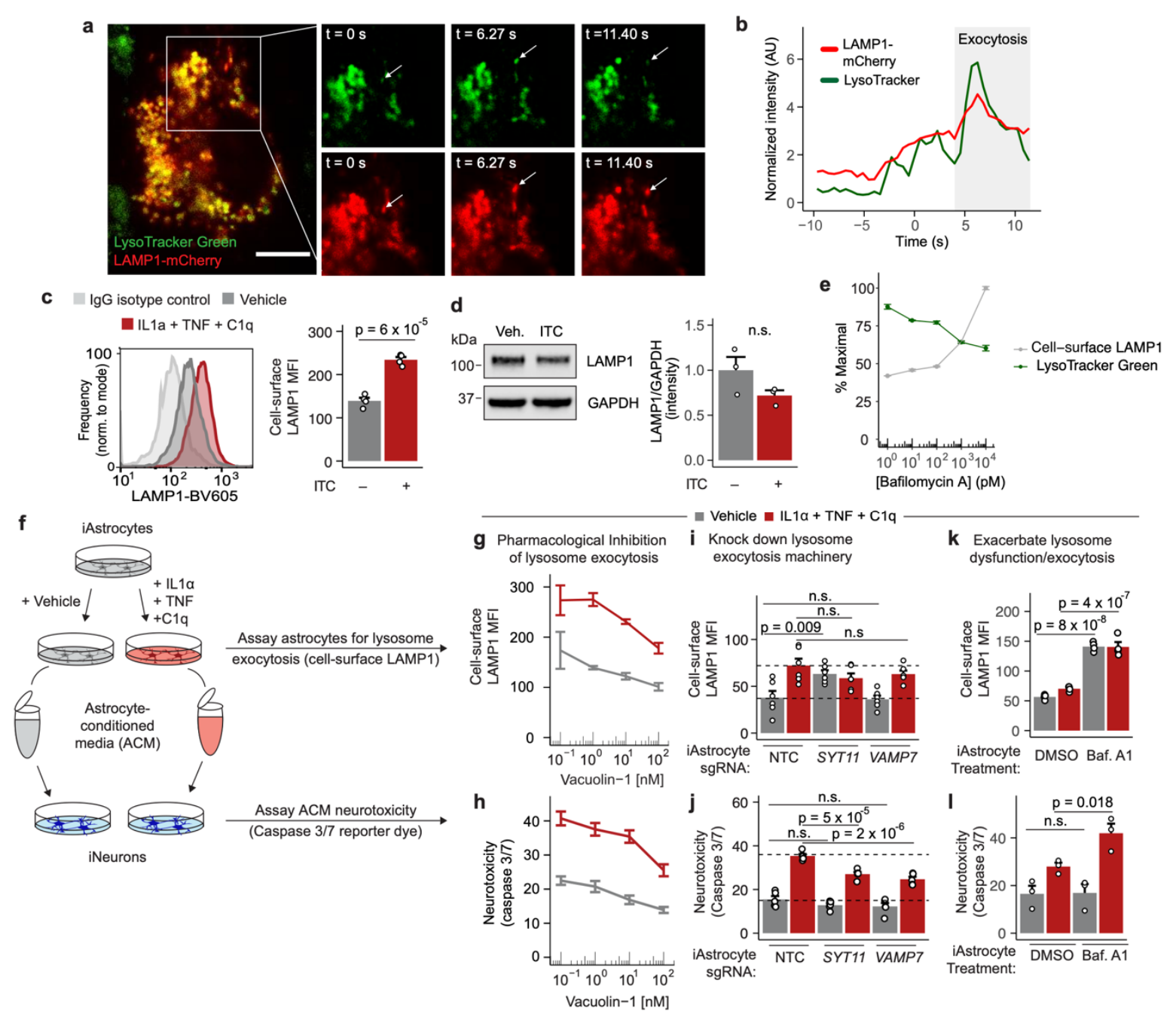

Fig. 2 | Lysosome exocytosis controls neurotoxicity of inflammatory reactive astrocytes. a, Representative TIRF microscopy images of a lysosome exocytosis event (arrows) in iAstrocytes. b, Quantification of LAMP1-mCherry (lysosomal membrane) and LysoTracker (lysosomal lumen) fluorescence intensity over the course of the lysosome exocytosis event depicted in a. c, Cell-surface LAMP1 for iAstrocytes treated with ITC or a vehicle control measured by flow cytometry. Left, representative flow cytometry results, including isotype control staining; right, Quantification of replicates, with the average median fluorescence intensity (MFI) value of IgG isotype controls ( $\mathrm{n}=2$ for each condition) subtracted from the LAMP1 MFI values $(n=4$ for each condition). d, Total LAMP1 protein in ITC and vehicle-treated iAstrocytes. Left, Representative immunoblot; right, quantification of independent wells ( $n=3$ for each condition). e, Bafilomycin A dose-response curves of LysoTracker and cell-surface LAMP1 measured by flow cytometry in iAstrocytes ( $n=3$ wells per condition). Values are quantified as the percent of the maximal value obtained in each individual experiment (LysoTracker or cell-surface LAMP1). f, Experimental strategy for parallel measurement of lysosome exocytosis and neurotoxicity of astrocyte conditioned media (ACM) (see Methods). g-l, Quantification of lysosome exocytosis in iAstrocytes (g, $\mathbf{i}$, and $\mathbf{k}$ ) and neurotoxicity of astrocyte (measured by 
caspase 3/7 activity reporter dye intensity) in iNeurons (h, $\mathbf{j}$, and $\mathbf{l})$ after treating iAstrocytes with the lysosome exocytosis inhibitor vacuolin-1 (g,h), knocking down lysosome exocytosis machinery in iAstrocytes with sgRNAs targeting SYT11 and VAMP7 (i,j), or treating iAstrocytes the lysosome exocytosis-inducing compound bafilomycin A (k,l). For bar graphs, data points represent individual wells with error bars representing the s.e.m. The two-sided Student's t-test was used in panels c-d and linear regression was used in panels i-l to compute p-values; for linear regression, p-values were corrected for multiple testing using Holm's method. 
bioRxiv preprint doi: https://doi.org/10.1101/2021.09.11.459904; this version posted September 12, 2021. The copyright holder for this preprint (which was not certified by peer review) is the author/funder, who has granted bioRxiv a license to display the preprint in perpetuity. It is made available under aCC-BY 4.0 International license.

a

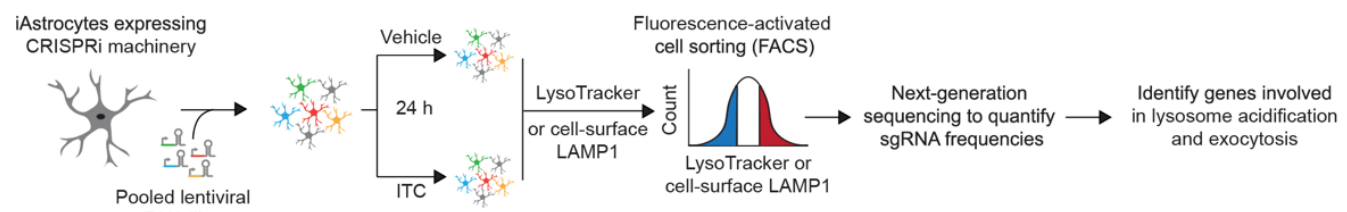

b
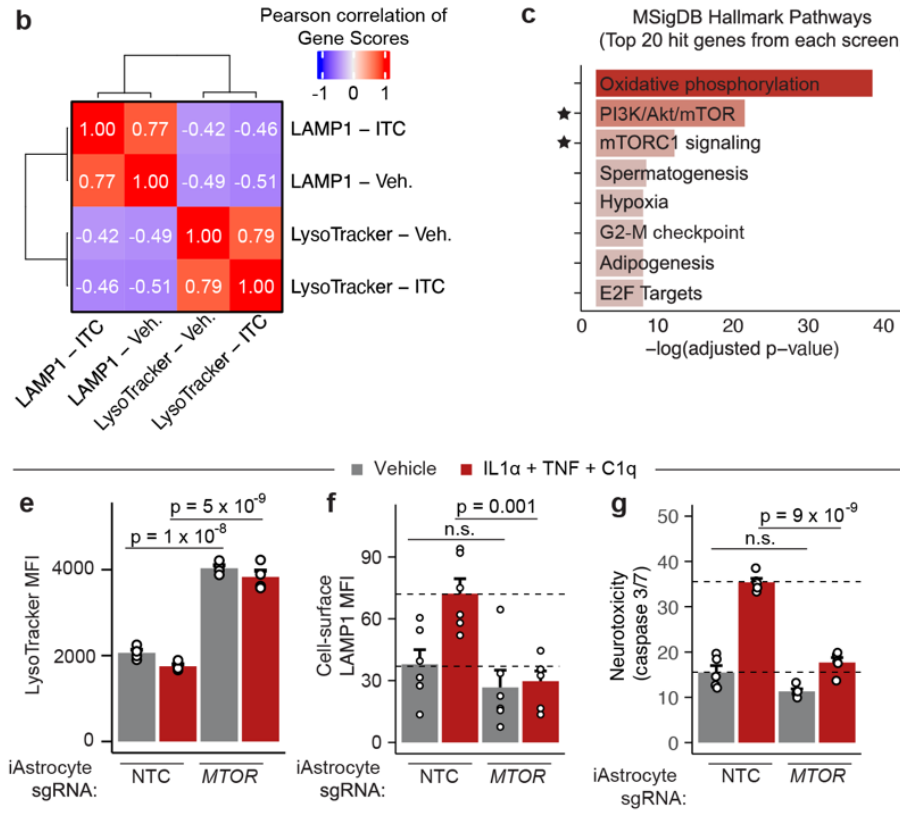

h

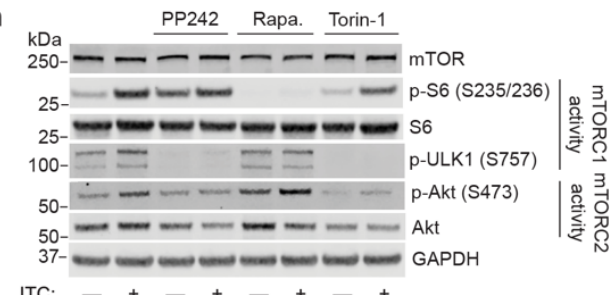

ITC: -+-++++
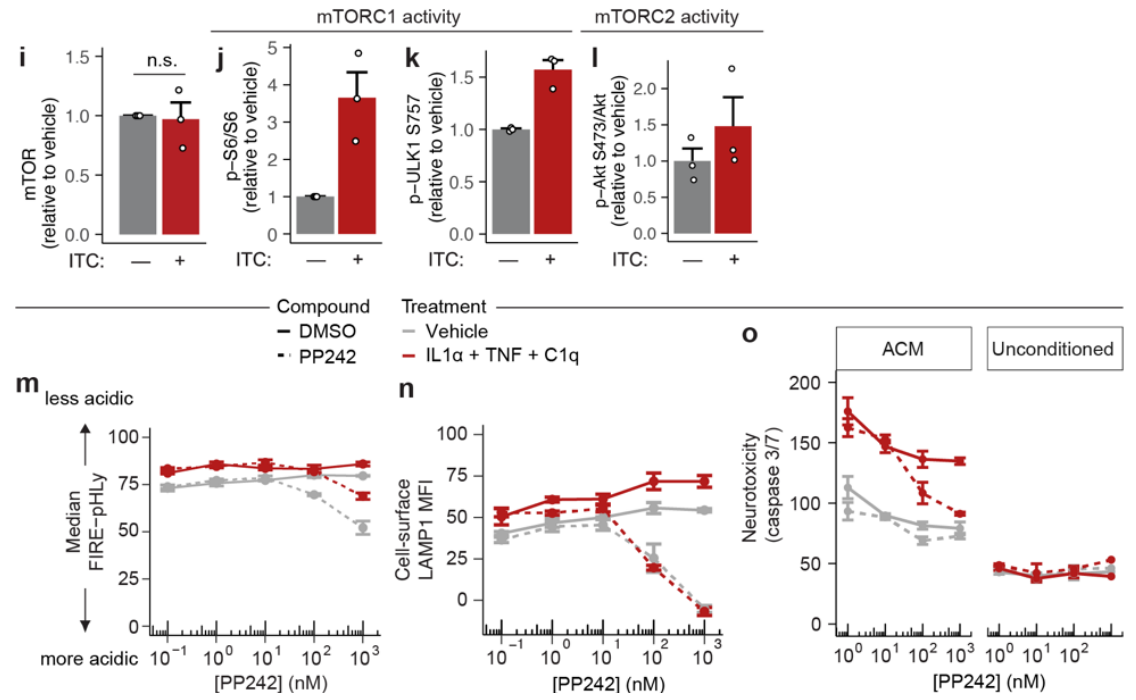

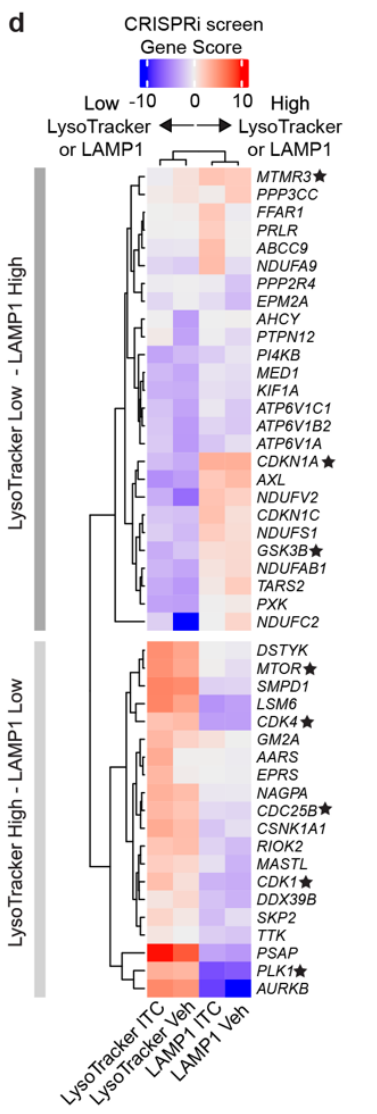

p

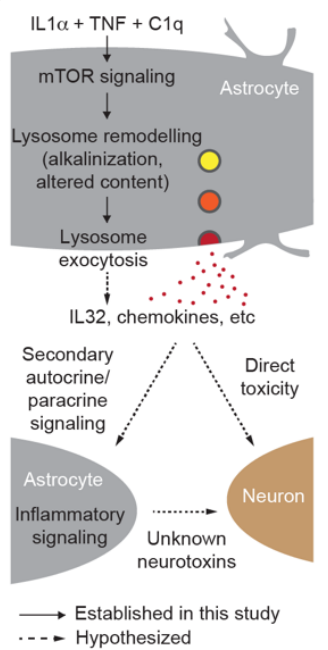

Fig. 3 (Legend overleaf) 
Fig. 3 | CRISPRi screens uncover mTOR as a regulator of lysosome exocytosis and inflammatory reactive astrocyte-mediated neurotoxicity. a, Workflow of FACS-based CRISPRi screens in iAstrocytes (see Methods). b, Pearson correlations of the gene scores (see Methods) of hit genes from the 4 CRISPRi screens completed ( $\mathrm{n}=2$ per condition). $\mathbf{c}$, Bar graph of Molecular Signature Database (MSigDB) terms whose constituent genes are most significantly enriched among the 20 strongest hit genes of any CRISPRi screen (46 genes total, see Methods). d, Heatmap of gene scores across all CRISPRi screens for genes from c, with mTOR-related hits starred. e-g, iAstrocytes were transduced with non-targeting control (NTC) or MTOR sgRNA and treated with IL1a + TNF + C1q (ITC) or vehicle as indicated $(\mathrm{n}=3$ independent wells per condition), and (e) LysoTracker staining (f) cell-surface LAMP1, and (g) neurotoxicity of astrocyte-conditioned media were quantified. $\mathbf{h}$, Representative immunoblots of mTOR levels and activity in iAstrocytes treated with a panel of mTOR inhibitors in combination with ITC or vehicle control. i-l, Quantification of immunoblots (from $n=3$ independent wells for each condition). m-o, Dose-response curves of (m) lysosome acidification (measured by flow cytometry of FIRE-pHLy-expressing iAstrocytes, (n) lysosome exocytosis (flow cytometry measurement of cell-surface LAMP1, and (o) astrocyte-mediated neurotoxicity (measured by caspase 3/7 activity reporter dye intensity) upon treatment of iAstrocytes with the pan-mTORC inhibitor PP242 in conjunction with vehicle vs. ITC ( $n=3$ independent wells for each condition). p, Model for a potential role of mTOR-dependent lysosomal remodelling and exocytosis in astrocyte-mediated neurotoxicity. For bar graphs, data points represent individual wells with error bars representing the s.e.m. Linear regression was used to compute p-values in all panels; p-values were corrected for multiple testing using Holm's method. 
a

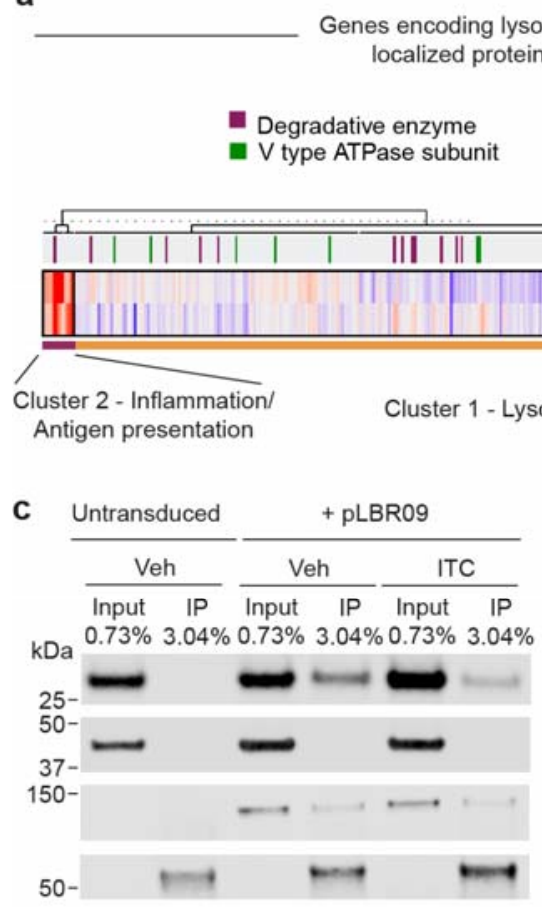

cathepsin D

$\beta$-actin

GFP

(PLBR09)

anti-mouse

(anti-HA antibody)

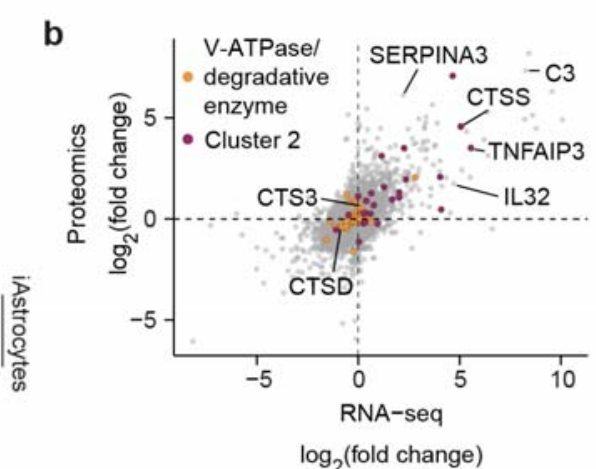

d

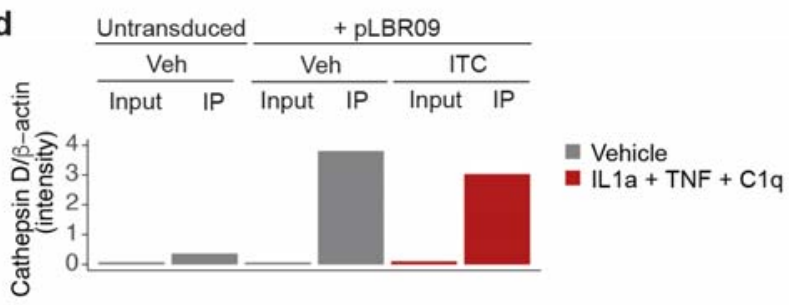

Extended Data Fig. 1 | Further characterization of lysosome remodeling in inflammatory reactive astrocytes. a, Heatmap of the mean $\log _{2}$ (fold change) (ITC-treated astrocytes relative to vehicle-treated astrocytes) of genes or proteins with GO annotations of lysosome, lysosomal membrane, or lysosomal lumen from RNA-seq or whole-cell proteomics. c, Representative immunoblot of vehicle and ITC-treated samples obtained for lysosome immunoprecipitation (lyso-IP) experiment (+pLBR09, IP) with untransduced and non-immunoprecipitated (+pLBR09, Input) negative controls. d, Quantification of the cathepsin D (lysosomal lumen to B-actin (cytosol) ratio from immunoblots shown in c. 

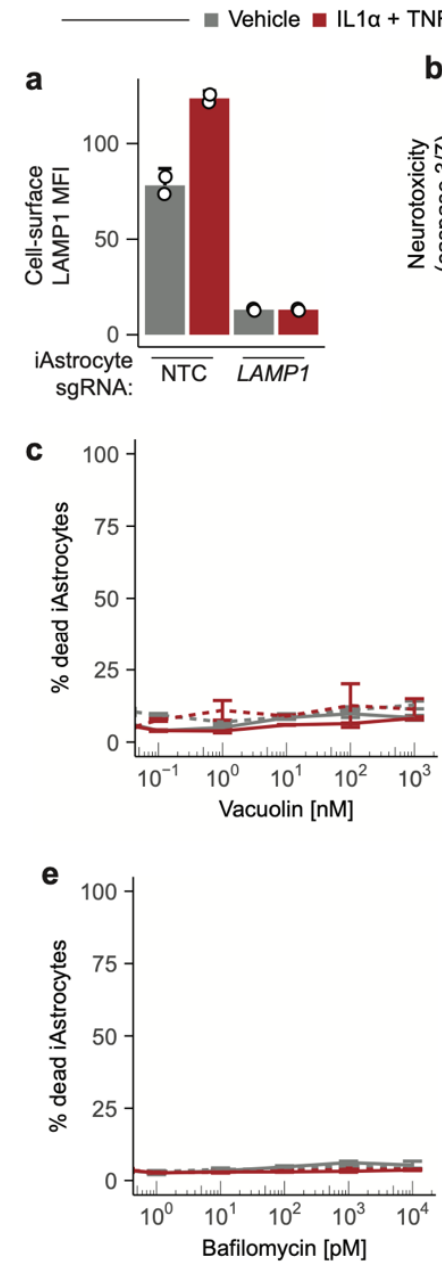

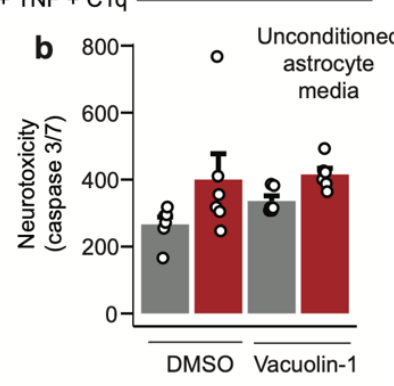

d

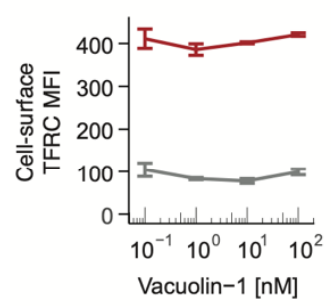

f

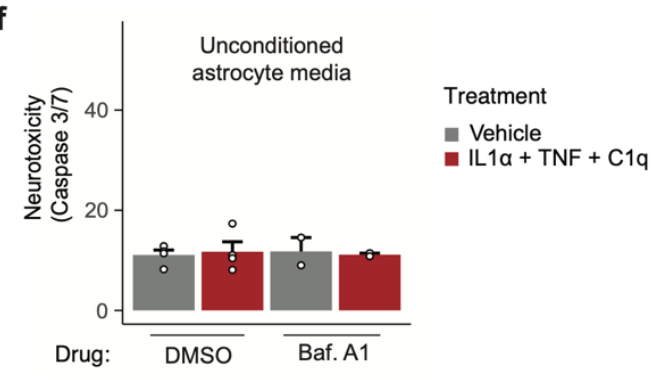

Extended Data Fig. 2 | Further characterization of the lysosome alkalinization-exocytosisneurotoxicity axis in iAstrocytes. a, Cell-surface LAMP1 levels (median fluorescence intensity - MFI; measured by flow cytometry) in vehicle and ITC-treated iAstrocytes transduced with NTC or LAMP1 sgRNA. b, Apoptosis of iNeurons treated with unconditioned astrocyte media containing the maximum concentration of vacuolin used in this study $(100 \mathrm{nM})$. c, Percentage of dead iAstrocytes (measured by flow cytometry as DAPI+ cells) in response to concentrations of vacuolin used in this study. d, Cell-surface TFRC levels (MFI; measured by flow cytometry) of the iAstrocytes shown in Fig. 2g,h. e, Percentage of dead iAstrocytes (measured by flow cytometry as DAPI+ cells) in response to concentrations of bafilomycin A used in this study. $\mathbf{f}$, Apoptosis of iNeurons treated with unconditioned astrocyte media containing the maximum concentration of bafilomycin A used in conditioned media experiments $10 \mathrm{nM}$ ). 

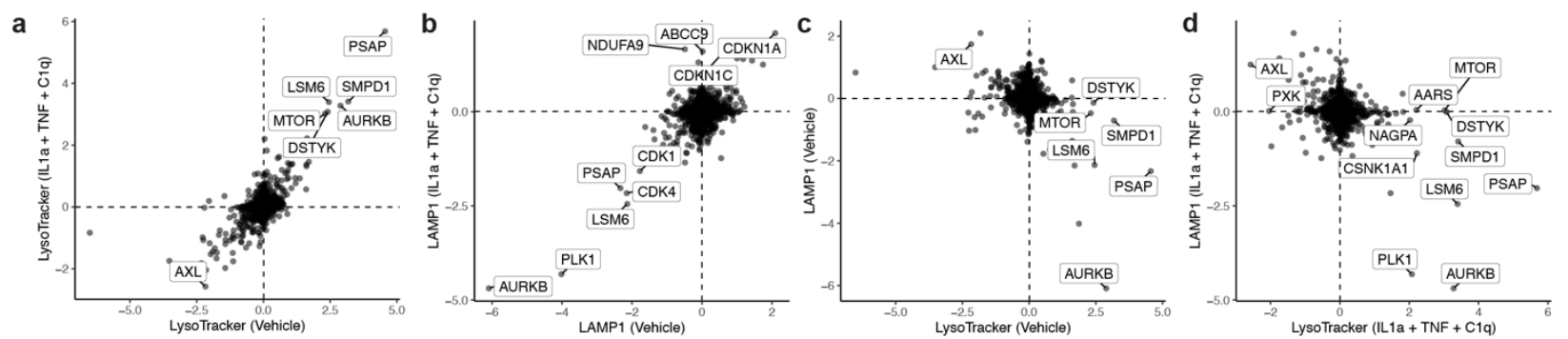

Extended Data Fig. 3 | LysoTracker and LAMP1 CRISPRi screening results. Scatterplots comparing gene scores (see Methods) between screens for (a) LysoTracker, (b) LAMP1, (c) vehicle-treated screens, and (d) ITC-treated screens. 


\section{SUPPLEMENTARY INFORMATION}
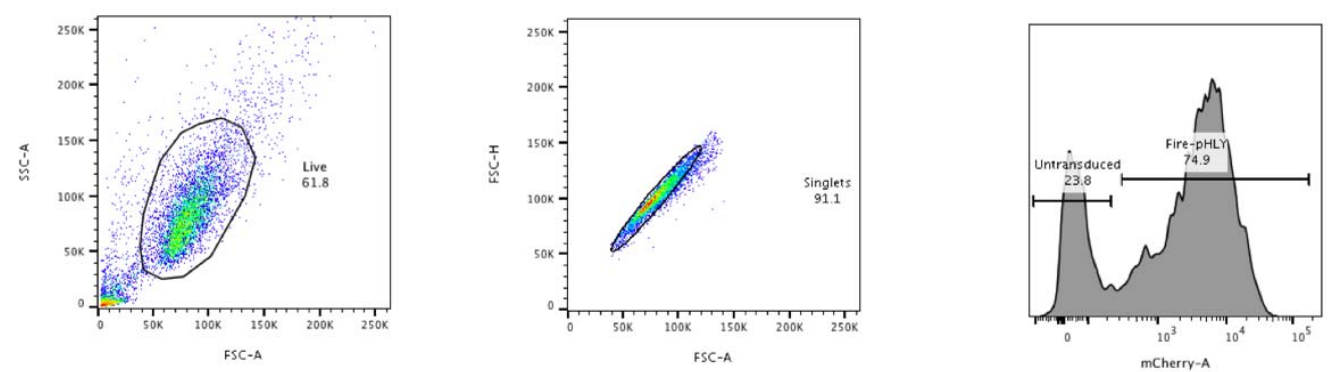

Supplementary Fig. 1 Gating strategy for general flow cytometry experiments (Live and Singlets gates) and FIRE-pHLy experiments (FIRE-pHLy gate).

a

Probe: anti-LAMP2 (top), anti-GAPDH (bottom)

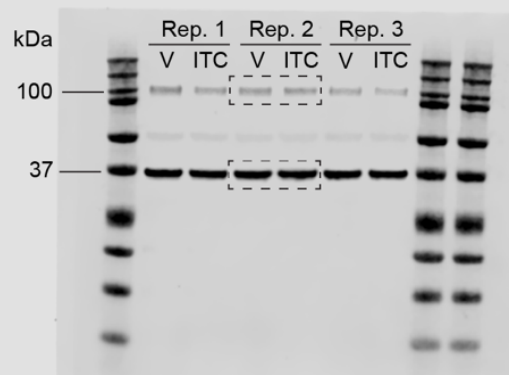

b

Probe: anti-LAMP1 (top), anti-MITF (bottom)

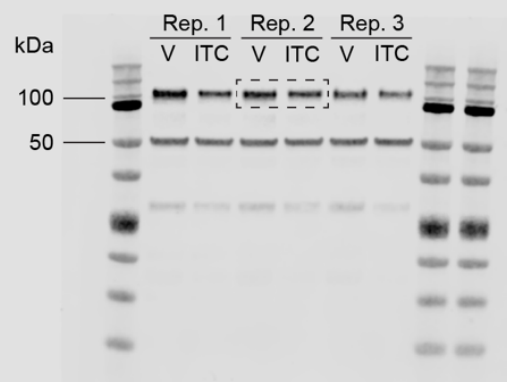

Supplementary Fig. 2 Source Data for (a) Fig. 1d and (b) Fig. 2d. 
bioRxiv preprint doi: https://doi.org/10.1101/2021.09.11.459904; this version posted September 12, 2021. The copyright holder for this preprint (which was not certified by peer review) is the author/funder, who has granted bioRxiv a license to display the preprint in perpetuity. It is made available under aCC-BY 4.0 International license.

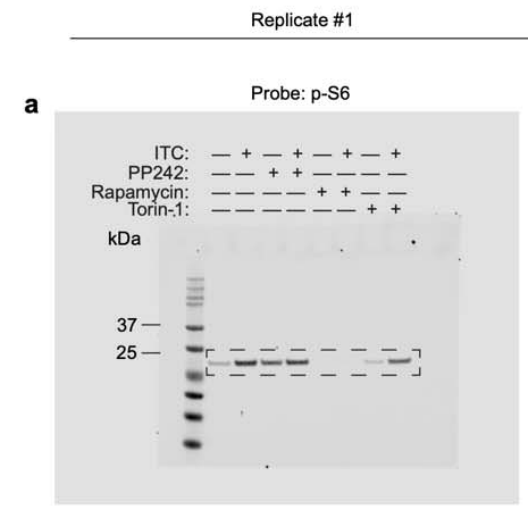

Ponceau staining

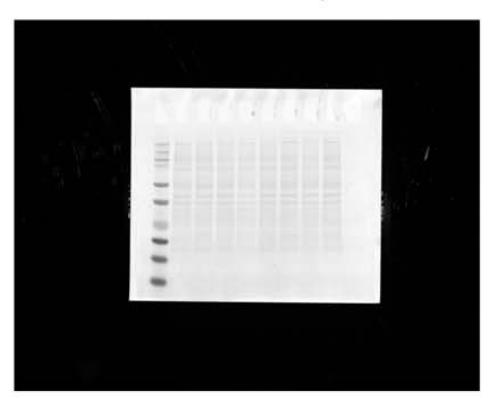

Probe: S6

b

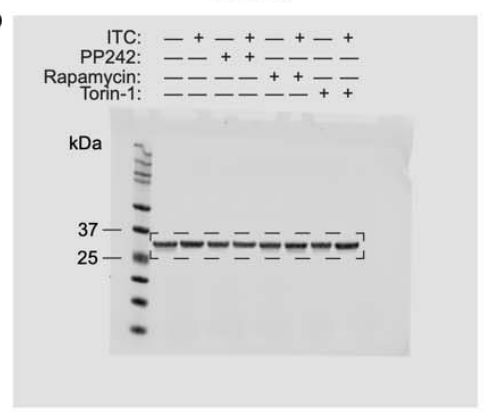

Ponceau staining

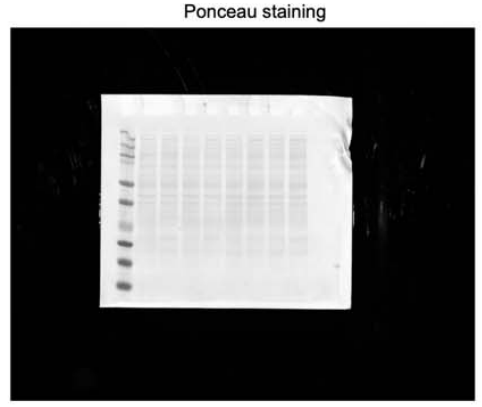

Replicate \#2

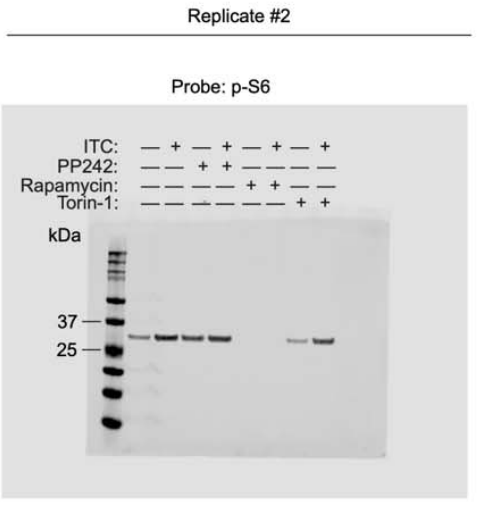

Ponceau staining

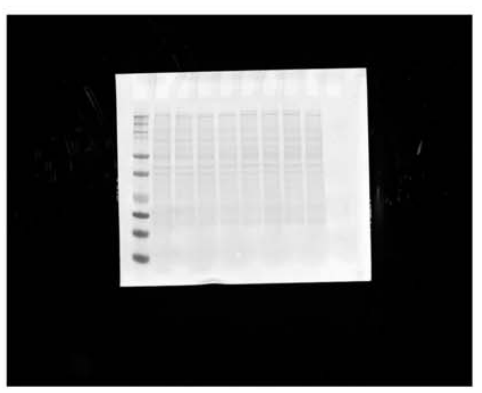

Probe: S6

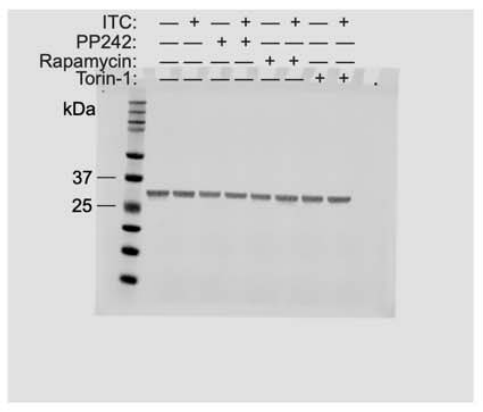

Ponceau staining

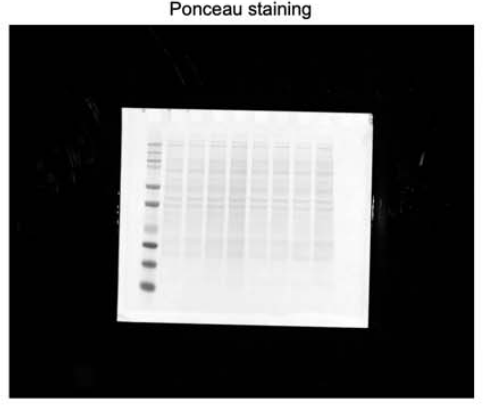

Replicate \#3

Probe: $\mathrm{p}-\mathrm{S} 6$

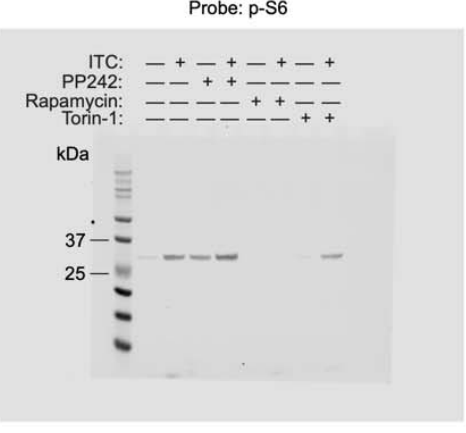

Ponceau staining

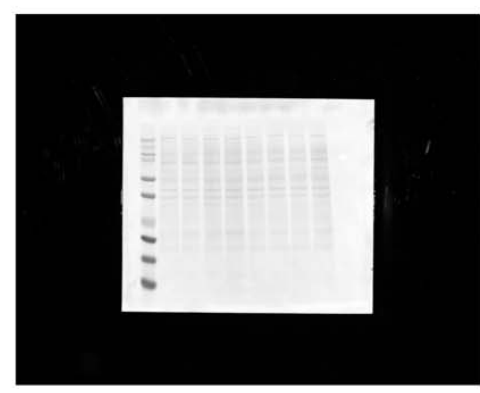

Probe: S6

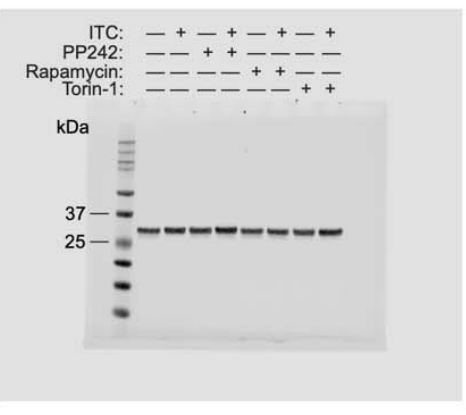

Ponceau staining

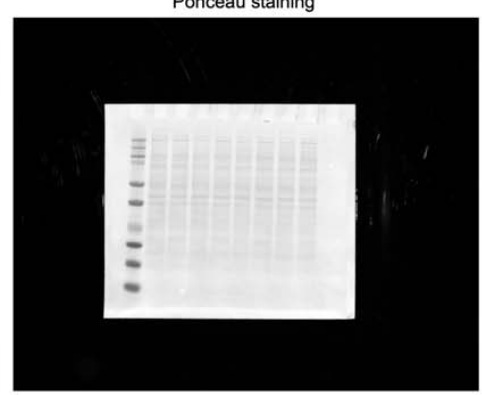

Supplementary Fig. 3 Source Data for Fig. 3h. 
bioRxiv preprint doi: https://doi.org/10.1101/2021.09.11.459904; this version posted September 12, 2021. The copyright holder for this preprint (which was not certified by peer review) is the author/funder, who has granted bioRxiv a license to display the preprint in perpetuity. It is made available under aCC-BY 4.0 International license.

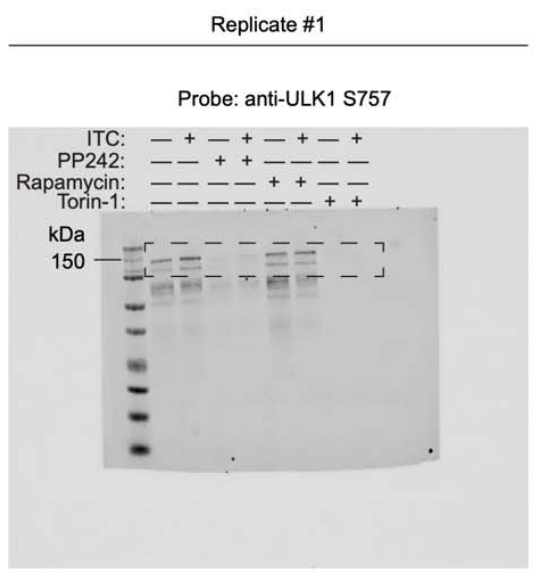

Probe: Akt

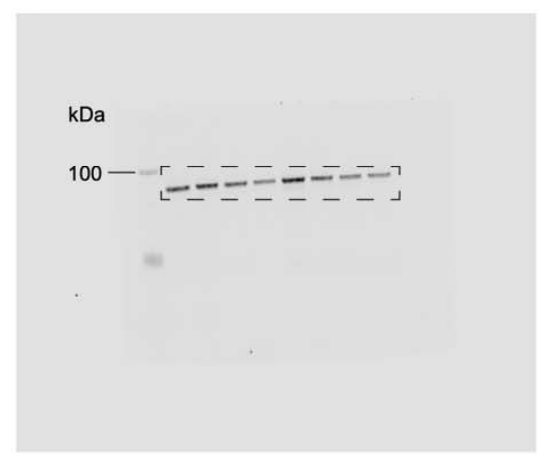

Ponceau staining

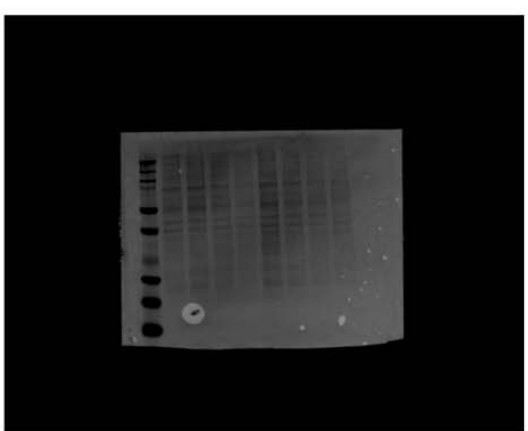

Supplementary Fig. 4 Source Data for Fig. 3h.
Replicate \#2

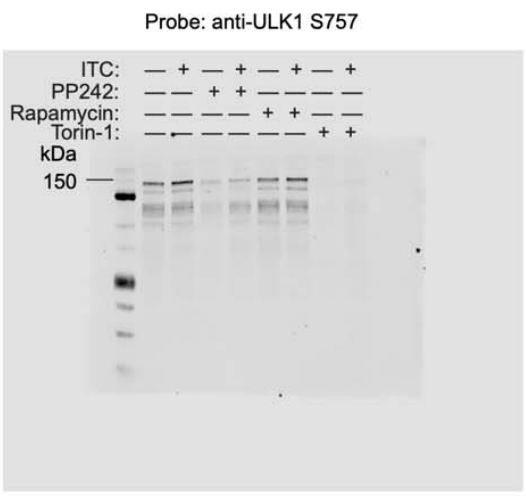

Probe: Akt

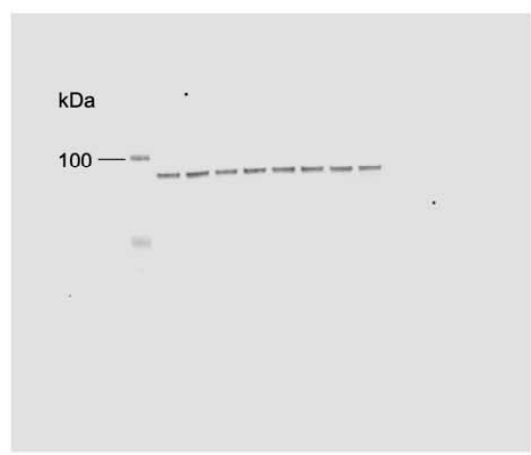

Ponceau staining

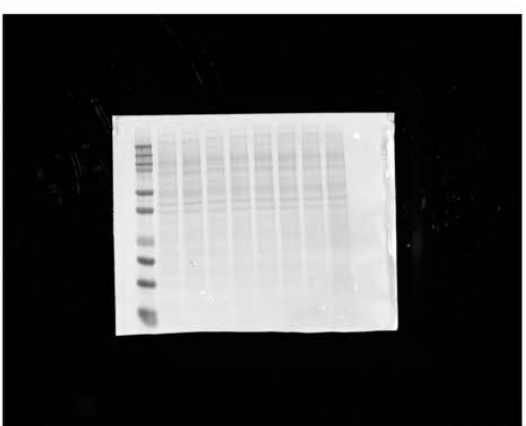

Replicate \#3

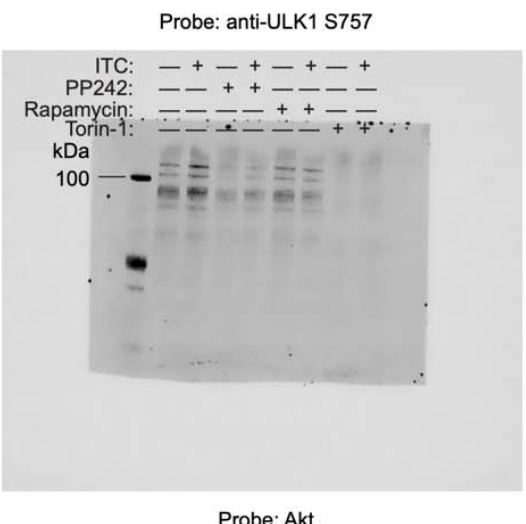

Probe: Akt

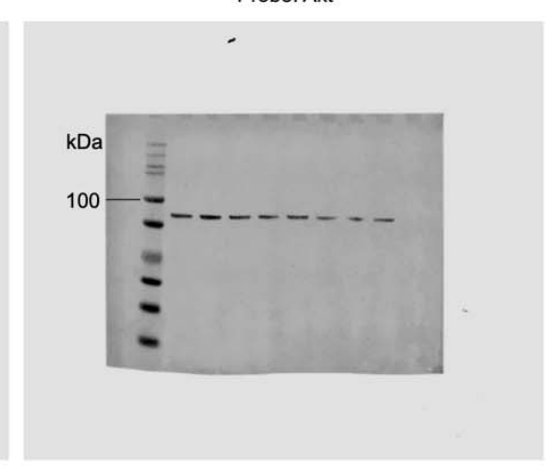

Ponceau staining

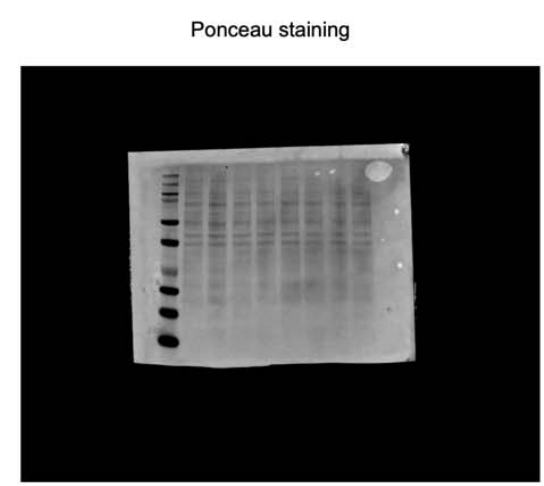


bioRxiv preprint doi: https://doi.org/10.1101/2021.09.11.459904; this version posted September 12, 2021. The copyright holder for this preprint (which was not certified by peer review) is the author/funder, who has granted bioRxiv a license to display the preprint in perpetuity. It is made available under aCC-BY 4.0 International license.

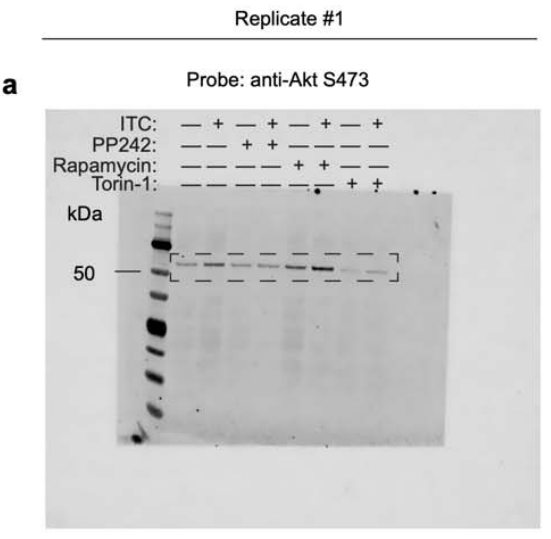

Ponceau staining

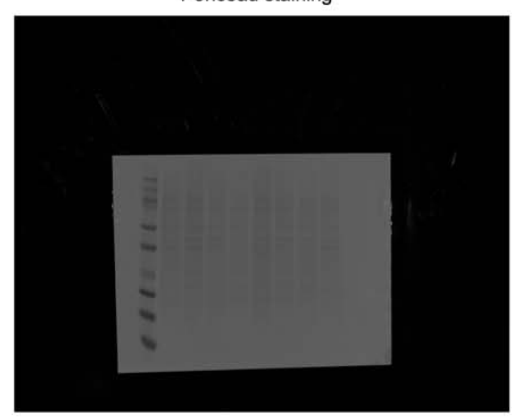

b

Probe: anti-MTOR (Rb, top); anti-GAPDH (Ms, bottom)

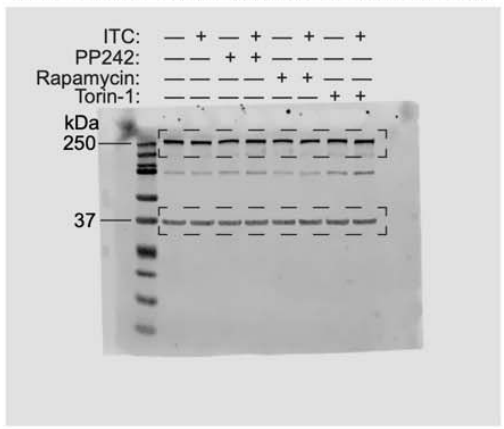

Ponceau staining

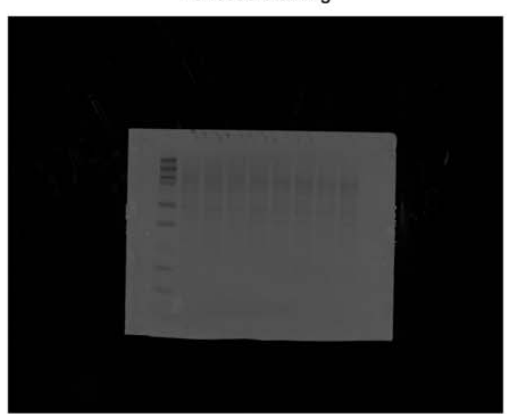

Replicate \#2

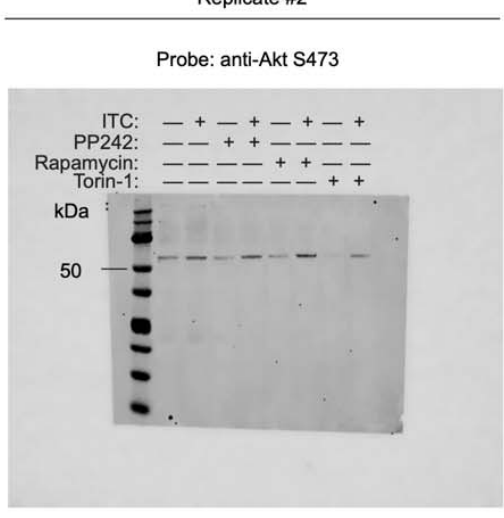

Ponceau staining

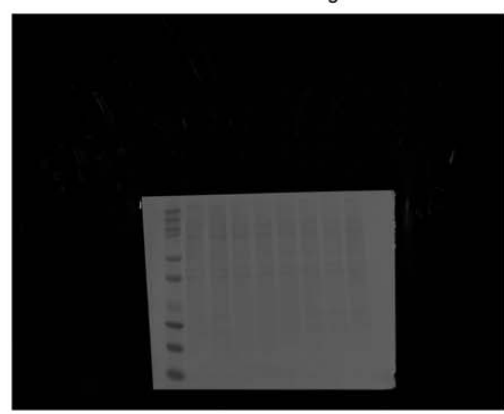

Probe: anti-MTOR (Rb, top); anti-GAPDH (Ms, bottom)

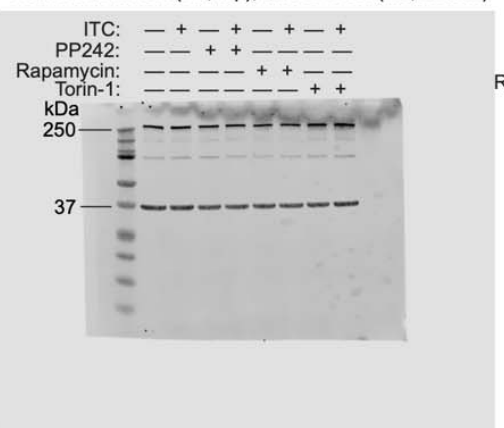

Ponceau staining

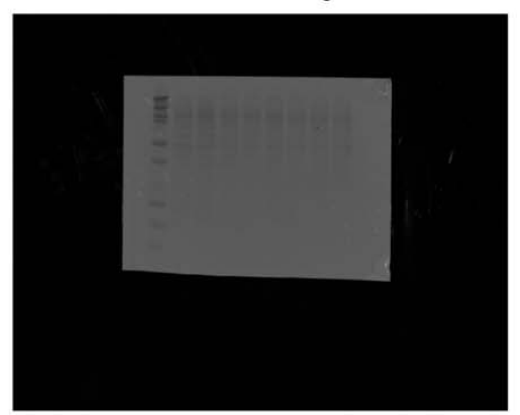

Replicate \#3

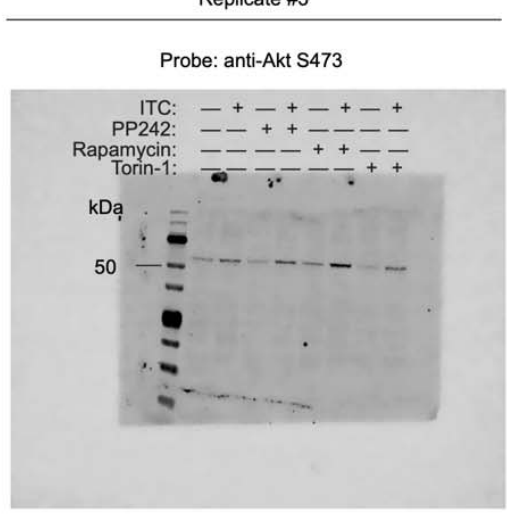

Ponceau staining

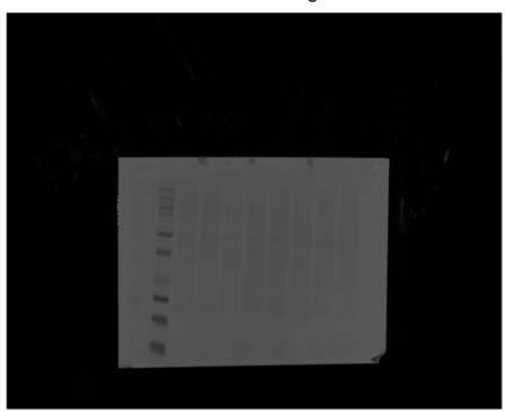

Probe: anti-MTOR (Rb, top); anti-GAPDH (Ms, bottom)

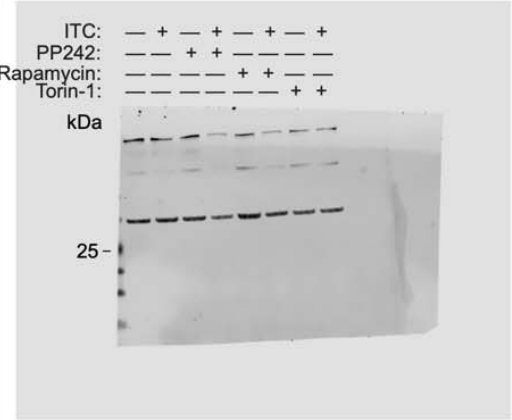

Ponceau staining

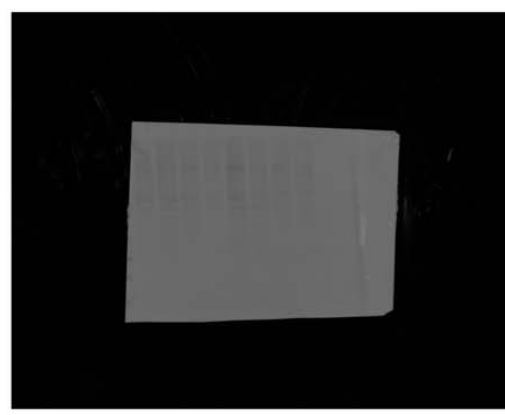

Supplementary Fig. 5 Source Data for Fig. 3h. 


\section{Supplementary Table Legends}

Supplementary Table 1 | Re-analysis of previously published hiPSC-derived astrocyte RNA-seq data. Differentially expressed genes (DEGs) induced by ITC in iAstrocytes and Krencik et $a l .{ }^{33}$ astrocytes were taken from Supplementary Table 1 from Leng et al. ${ }^{5}$. DEGs induced by ITC in human cerebral organoid-derived astrocytes from Barbar et al. ${ }^{4}$ and DEGs induced by IL-1 $\beta+$ TNF in hiPSC-derived astrocytes from Perriot et al. ${ }^{8}$ were called by analyzing the raw data from those studies with BioJupies ${ }^{28}$.

Supplementary Table 2 | Whole-cell and lyso-IP proteomics. "LFQ intensity": $\log _{2}$-scaled sum of label-free quantification intensities from all peptides within the protein group from

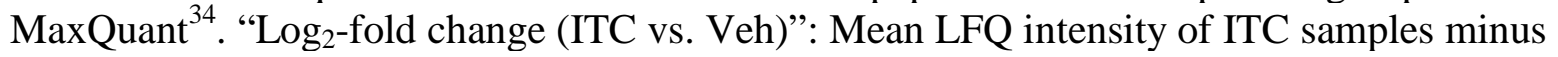
mean LFQ intensity of Veh samples. "Log-scaled p-value": - $\log _{10}(\mathrm{t}$-test p-value). "FDR (qvalue)": permutation-based false discovery rate calculated from t-test p-values 35 . "Majority protein IDs": UniProtKB IDs of proteins that have at least half of the peptides that the leading protein has.

Supplementary Table 3 | Phenotype scores and p-values from CRISPRi screens. Gene_TSS: gene name followed by the transcriptional start site (TSS) targeted by sgRNAs for genes with more than one TSS. Phenotype score: log-scaled fold difference between sgRNA abundance in high bin vs. low bin. Gene score: $-\log _{10}(P$ value $) *($ Phenotype Score $)$.

\section{Supplementary Movie Legends}

Supplementary Movie 1 | Visualization of iAstrocyte lysosomes via TIRF microscopy. Timelapse movie (30 fps) of iAstrocytes stained with LysoTracker Green (green, lysosome lumen) and transduced with FIRE-pHLy (red, lysosome membrane) (see Methods).

\section{Supplementary Movie 2 | Visualization of individual lysosome exocytosis events in} iAstrocytes. Time-lapse movie (5 fps) of highlighted area in Supplementary Movie 1. Arrow highlights a putative lysosome exocytosis event. 